\title{
DISTRIBUTED CONTROL TO IMPROVE PERFORMANCE OF \\ THERMOELECTRIC COOLERS
}

\author{
By \\ Richard D. Harvey \\ Thesis \\ Submitted to the Faculty of the \\ Graduate School of Vanderbilt University \\ in partial fulfillment of the requirements \\ for the degree of
}

MASTER OF SCIENCE
in
Mechanical Engineering

August, 2005

Nashville, Tennessee
Approved:
D.G. Walker
K.D. Frampton
A.M. Strauss 
To little Sarah, you are truly a miracle. 


\section{ACKNOWLEDGEMENTS}

I would like to express my gratitude to the United States Navy for providing me the opportunity and financial support to attend a fine university such as Vanderbilt. This duty assignment and life experience has been incredibly rewarding.

Also, I'd like to thank the members of my thesis committee for professional guidance and advice throughout this endeavor. I especially appreciate the guidance provided by Professor Greg Walker. This work would not have been possible without his limitless encouragement and insight.

No one is more important to me than my remarkable family. I am grateful to my parents and grandparents for providing a lifetime of loving support. Finally, thanks to my darling wife, Kelley, and my three wonderful children, Stephanie, Joshua, and Sarah who have always enthusiastically supported my education and provided unending inspiration. 


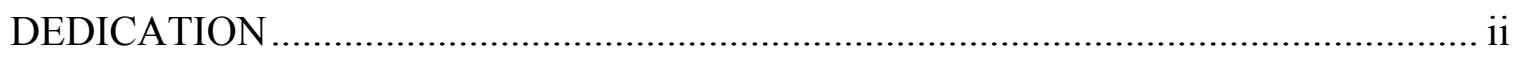

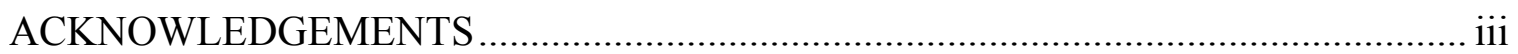

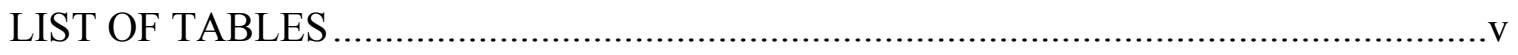

LIST OF FIGURES ………………………………....................................... vi

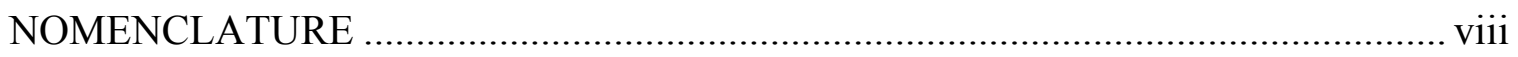

Chapter

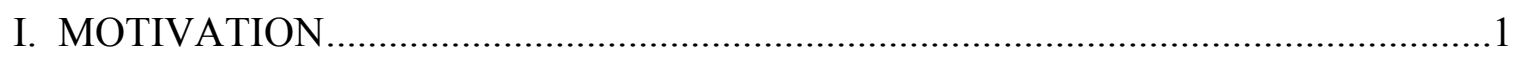

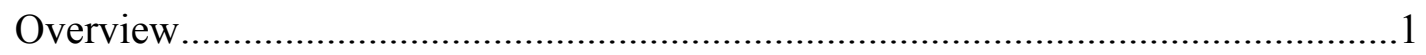

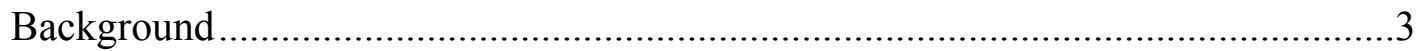

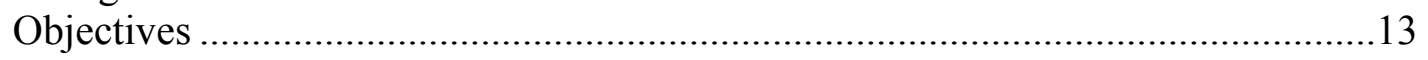

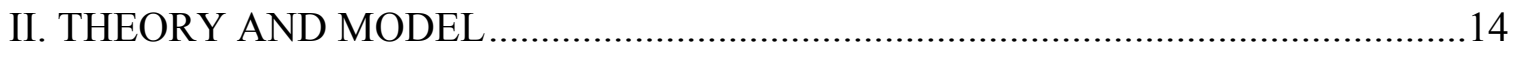

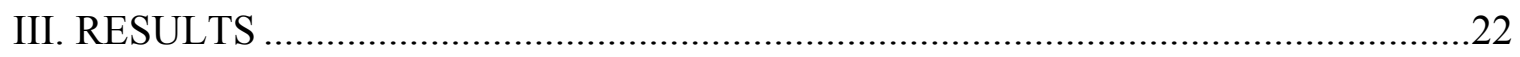

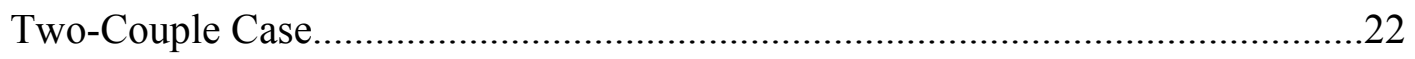

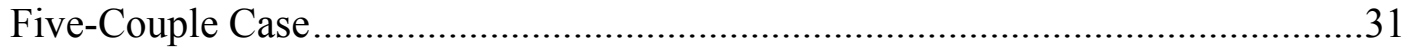

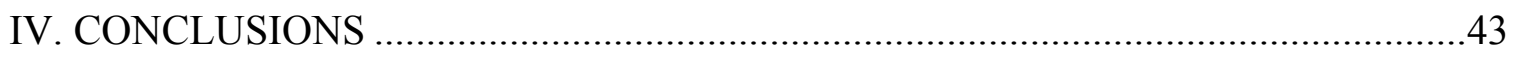

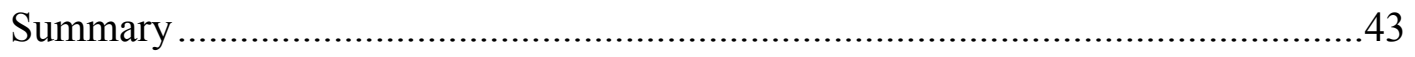

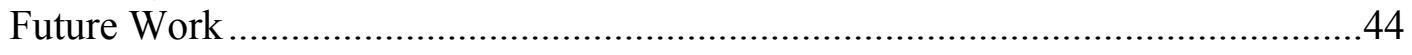

Appendix

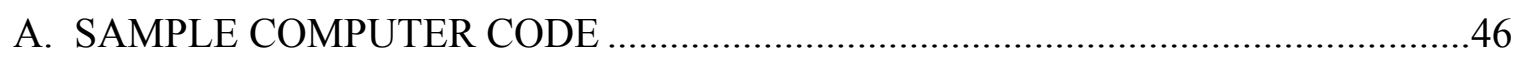

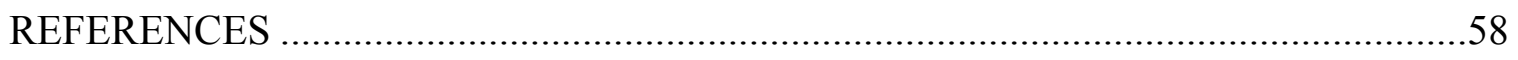




\section{LIST OF TABLES}

Table

Page

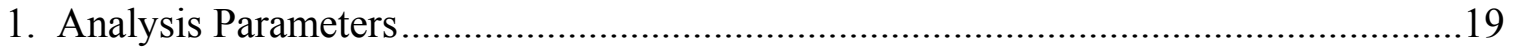




\section{LIST OF FIGURES}

Figure

Page

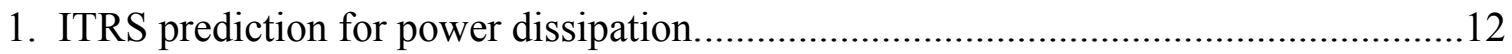

2. Schematic of a typical thermoelectric cooling element ...........................................14

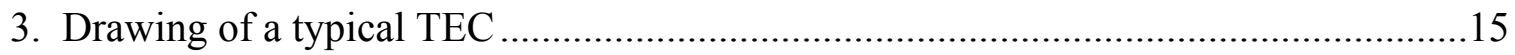

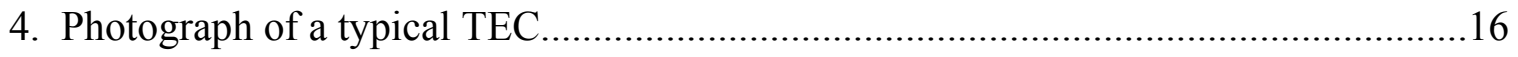

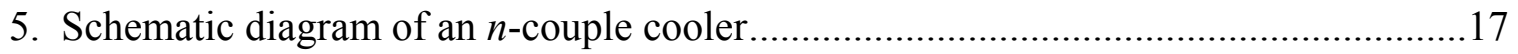

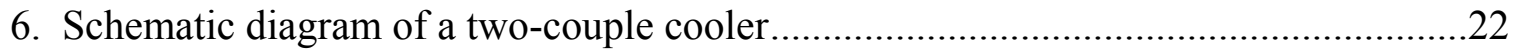

7. Junction temperature vs. current for a constant heat load......................................23

8. Coefficient of performance vs. current for a constant heat load...............................24

9. Coefficient of performance vs. current for a constant heat load (cropped) .................25

10. Comparison of independent operation and simultaneous operation for current vs. the amount of heat removed.....

11. Comparison of independent operation and simultaneous operation for junction temperature vs. the amount of heat removed.

12. Comparison of independent operation and simultaneous operation for $C O P$ vs. the amount of heat removed (high lateral conductivity) ....

13. Comparison of independent operation and simultaneous operation for $C O P$ vs. the amount of heat removed (low lateral conductivity)

14. Comparison of serial, optimum and rule-based values for $C O P$ vs. the amount of heat removed.

15. Comparison of optimum and rule-based values for $T_{h}$ vs. current using multiple linear regression analysis.

16. Comparison of optimum and rule-based values for $T_{h}$ vs. current using multiple polynomial regression analysis. 
17. Comparison of optimum and rule-based values for $T_{h}$ vs. current using multiple polynomial regression analysis, $T_{h}$-squared term only

18. Comparison of optimum and rule-based values for junction temperature, $T_{j 1}$ vs. heat removed

19. Comparison of optimum and rule-based values for junction temperature, $T_{j 2}$ vs. heat removed

20. Comparison of heat load assumption for each thermocouple

21. Comparison of optimum and serial values of $C O P$ for different values of lateral thermal conductivity,

22. Comparison of serial, optimum and rule-based values of $C O P$ for different values of equivalent thermal conductivity, $R_{2}$. 


\section{NOMENCLATURE}

$$
\begin{aligned}
& \text { Symbol - Meaning } \\
& \alpha-\text { Seebeck coefficient } \\
& \pi \text { - Peltier coefficient } \\
& \tau-\text { Thomson coefficient } \\
& \rho \text { - electrical resistivity } \\
& \sigma-\text { electrical conductivity } \\
& k-\text { thermal conductivity } \\
& V \text { - voltage } \\
& I \text { - current } \\
& T \text { - temperature } \\
& Z \text { - figure of merit } \\
& Z T \text { - dimensionless figure of merit } \\
& C O P \text { - coefficient of performance } \\
& \Delta T \text { - temperature difference } \\
& T_{c} \text { - cold-side temperature } \\
& T_{h} \text { - hot-side temperature } \\
& T_{j} \text {-junction temperature } \\
& T_{a} \text {-ambient temperature } \\
& G \text {-geometric factor }
\end{aligned}
$$$$
\text { Symbol - Meaning }
$$$$
W \text { - amount of work }
$$$$
n \text { - number of couples }
$$$$
i \text {-a specific numbered couple }
$$$$
Q \text { - amount of heat }
$$$$
Q_{g e n}-\text { amount of heat generated }
$$$$
Q_{\text {cond }} \text { - amount of heat conducted }
$$$$
R \text { - resistance to heat transfer }
$$$$
R_{\text {cond }} \text { - lateral thermal resistance }
$$$$
\mathrm{d} T \text { - differential temperature }
$$$$
\mathrm{W}-\text { watt }
$$$$
\text { A - amperes }
$$$$
\text { K - Kelvin }
$$$$
{ }^{\circ} \mathrm{C} \text { - degrees Celsius }
$$$$
\mathrm{m} \text { - meter }
$$$$
\mathrm{cm}-\text { centimeter }
$$$$
\Omega-\text { ohm }
$$$$
\dot{Q} \text { - heat rate }
$$$$
k_{\text {cond }}-\text { lateral thermal conductivity }
$$ 


\section{CHAPTER I}

\section{MOTIVATION}

\section{Overview}

Although many research efforts to find alternative miniature refrigerators exist, thermoelectric coolers (TECs) are currently the only miniature cooling devices finding widespread use in microelectronics cooling applications [1]. Unlike other miniature cooling schemes, TECs are solid-state devices and, therefore, are reliable, compact, and quiet. However, TECs generally suffer from poor device efficiencies, which limit their widespread applicability. To overcome these performance limitations, many research initiatives are currently seeking to improve the effectiveness of TECs by improving the materials used for these coolers [2]. Examples of possibilities that are being explored include skutterudites, nanotechnology, superlattices, and thin-films [3-8]. Additional research has also been devoted to improving fin-fan systems, minimizing contact resistances, and employing multistage TECs, which have already been implemented in chip-cooling applications [9-14].

The typical TEC unit that is currently marketed for chip-cooling applications consists of many individual thermocouples, called elements or cells, connected electrically in series, but thermally in parallel. Each individual thermocouple is composed of two dissimilar materials, called pellets or legs, one of which is usually a ptype semiconductor and the other an n-type semiconductor [15]. When the TEC unit is powered, all individual elements are powered simultaneously. 
In electronic chip cooling applications, the packaging consists of the chip, a TEC, a heat spreader, and finally, a fin-fan system. All of the contacts are enhanced with a thermal interface material (TIM). The heat spreader uniformly distributes the heat load produced by the chip. A TEC that is comparable in size and shape is contacted with the spreader to remove the heat away from the chip. The fin-fan system must still remove the heat from the TEC and away from the computer. The purpose of the TEC is to lower the processor temperature so that the integrated circuits may operate in a desired temperature band. Performance of modern computer chips will degrade above $85^{\circ} \mathrm{C}[16]$.

The general measure of efficiency of a TEC is based on the amount of heat that it removes compared to the amount of work that it requires. This value is referred to as coefficient of performance, $C O P$.

$$
C O P=\frac{Q_{\text {out }}}{W_{\text {in }}}
$$

The material effectiveness of a TEC is rated by a dimensionless figure of merit, $Z T$. Typical values for $Z T$ available from manufacturers are slightly less than unity. $Z T S$ required to cool the future generations of computer chips will need to approach three and eventually even higher [17]. This figure of merit is based solely on material properties, but does have a direct affect on the $C O P$ of the device. Recent material improvements have resulted in $Z T$ increases of between two-fold and three-fold [18]. Some sources purport theoretical increases even higher [19]. One problem with the current material improvements, however, is that they may require years to become fully implemented into industry.

One alternative approach that has only been recently considered is a method to control individual elements of the TEC separately based on their respective heat loads 
[20]. Because computer chips generate hot spots corresponding to the portion of the chip that is performing calculations at any given time, the heat load is not uniformly distributed. Heat spreaders improve the distribution of heat but do not transfer heat instantaneously [21]. Existing TECs can only be powered as a unit; all the elements receive the same amount of current regardless of their specific heat lead. Intuitively, it makes sense that $C O P$ could be improved if each TEC element is only powered when it is needed; and then, powered only to the degree that is required to remove the amount of heat being produced. Therefore, we propose to enhance the system efficiency by clever control of TEC units.

Distributed control refers to a method that allows the individual elements of a system to respond individually based on their respective state [22-23]. Previous research has shown the potential of increased effectiveness by separately controlling individual thermocouples or groups of thermocouples in TECs [24-25]. Another major benefit of distributed control is that it may be applied concurrently with other technological advances. Regardless of the material advances in TECs, distributed control would allow for the optimization of $C O P$. This paper strives to perform analysis for the basis of a strategy using distributed control to improve the performance of TECs.

\section{Background}

In 1821, Thomas Seebeck discovered the first of three thermoelectric effects by heating a junction of two dissimilar metals and producing a voltage [26]. In 1834, Jean Peltier discovered that by passing a current through a junction of two dissimilar metals, a temperature difference could be produced [27]. When the current is passed in one 
direction there is a cooling effect at one end. When the current direction is changed, the cooling occurs at the opposite end. The Seebeck effect and the Peltier effect were eventually mathematically derived by William Thomson, later referred to as Lord Kelvin, who also predicted a third thermoelectric effect [28]. The Thomson effect refers to the effect of passing a current through a single conductor with a temperature gradient thereby absorbing or releasing heat.

These thermoelectric effects are quite different than Joule heating, which is power lost from passing current through a conductor and is a function of electrical resistance and the amount of current. Contrarily, the thermoelectric effects are closely related to each other and can be exploited to produce power and refrigeration. Each of the effects has its own associated material property. The Seebeck coefficient, $\alpha$ is defined by

$$
\alpha=V / \Delta T
$$

where $V$ is the voltage produced and $\Delta T$ is the difference in temperature. The Peltier coefficient, $\pi$ is given by

$$
\pi=\dot{Q} / I
$$

where $\dot{Q}$ is the heating rate at one end of the conductor (cooling rate at the other end) and $I$ is the current passed through the conductor. The Thomson coefficient, $\tau$ is given by

$$
\tau=T d \alpha / d T
$$

where $d \alpha$ is Seebeck coefficient differential and $d T$ is the temperature differential. From (2)-(4), the relationship between the Seebeck and Peltier coefficients can be derived and is given by

$$
\pi=\alpha T
$$


Although the prospects for thermoelectric coolers were well understood, early efforts showed benefits to be minimal because of material limitations. Altenkirch in 1911 showed that a good thermoelectric material would have a high Seebeck coefficient, a high electrical conductivity and a low thermal conductivity [29]. In metals, the electrical conductivity and the thermal conductivity are closely related; therefore, alternate materials are needed. Not until semiconductors became mass-produced in the 1950s for transistor manufacturing did the value of thermoelectrics become realized. The desired quality in semiconductors is that the relationship between electrical and thermal conductivities starts to diverge. Doping levels further enhance this quality.

The parameter that is most used to measure a thermoelectric material's suitability is the thermoelectric figure-of-merit, $Z$, given by

$$
Z=\frac{\alpha^{2}}{\rho k},
$$

where $\rho$ is the electrical resistivity, $k$ thermal conductivity, and $\alpha$ is the Seebeck coefficient. From this relationship Altenkirch's findings are apparent. A high Seebeck coefficient (or Peltier coefficient) is desired in order to maximize the amount of heat transferred for the current applied. Less intuitive is the need for a low thermal conductivity and a low electrical resistivity. A low thermal conductivity is required to minimize parasitic heat transfer. This is heat flow in the direction opposing the desired direction of heat transfer due to a favorable temperature gradient. In other words, in a TEC heat will flow from the cold side to the hot side due to the thermoelectric effect. However, Fourier heat transfer will always influence heat to flow from the hot side to the cold side and will result in reduced performance of the TEC. Furthermore, a low electrical resistivity is required to minimize Joule heating. Joule heating produces extra 
heat internal to the TEC, again reducing the net amount of heat that the TEC can transfer. Because $Z$ varies with temperature, a dimensionless figure-of merit, $Z T$ is often used to describe a material's thermoelectric suitability.

In the 1930s, before the widespread application of semiconductor materials, typical estimates of $Z T$ were on the order of $0.2[19,30]$. This number is considerably low by today's standards and corresponds to an efficiency of about $4 \%$. Efficiency is the term often used to describe the benefit gained from TECs. However, efficiency is more accurately termed $C O P$, coefficient of performance and is given by (1), although the two terms are often used interchangeably [1]. ZT is strictly a material property, whereas $C O P$ is a measure of an entire system's ability to remove heat. $C O P$ is, however, directly influenced by $Z T$. For instance, a system that improves its material's $Z T$ without changing any other parameter will have a directly proportional result on $C O P$.

Once semiconductor materials became more prevalent in the $1950 \mathrm{~s}$, their thermoelectric properties were explored. Initially, the primary prospect for these materials was considered to be their thermoelectric capability and not their potential for use in integrated circuits [17]. ZTs increased steadily over the next decade up to about 1.0 for bismuth telluride, $\mathrm{Be}_{2} \mathrm{Te}_{3}$ [30]. Material advances slowed significantly after that and ZTs in the 1990s were still only around 1.0 [31]. The most common TEC semiconducting material currently in use is still bismuth telluride [32]. Due to improvements in manufacturing and better consistency in the bulk properties, bismuth telluride now has a nominal $Z T$ of about 1.2. This corresponds to a maximum $C O P$ of about 1.0. But even with these material advances, H. J. Goldsmid, postulates that the 
typical, commercially available TEC still has an effective $Z T$ of only about 0.8 [32]. This deficiency is due to difficulties and inconsistencies in manufacturing the TEC itself.

Interest in thermoelectric material research was reborn in the 1990 s due to increased applications prospects provided by the electronics boom, as well as advances in materials manufacturing processes [2]. TECs found more widespread use in device electronics cooling, such as cooling batteries and processors in cellular phones and portable video recorders. Currently, many research efforts are in progress to improve the performance of TECs. A very large percentage of these efforts concentrate on material improvement alone. Some efforts concentrate on improving the bulk semiconductor materials, although a theoretical $Z T$ limit of 2.0 has been theorized for these materials. The interest stems from the fact that so many of these materials have not been tested [32]. Basic thumb rules have been used in the past to narrow the field of materials to be tested [33]. Often times, though, the tests do not produce the expected results. Thus leaving the possibility that some, as of yet, unconsidered material will out perform the best thermoelectric semiconductors.

Other materials research efforts have concentrated on the new prospects that are now possible with nanoengineering or nanotechnology. Nanoengineering literally refers to the ability to engineer on the nanometer scale. This term implies the ability to design and create materials atom by atom. This field is further subdivided into several branches although the ultimate objective of each branch is quite similar. This objective is to create the diverging electrical and thermal conductivities. The theoretical ZT limits for these types of material are now estimated to be around 6.0 and beyond [33]. These predicted limits even exceed those necessary to rival a typical kitchen refrigerator or home air 
conditioner and would be adequate for chip cooling over the next decade [34].

Preliminary results strongly support the validity of the theoretical limits, although application and mass production of such materials are years away. Actual $Z T$ s produced in the lab only approach 2.0 at room temperature; nevertheless, this leap in $Z T$ is larger than all the others combined over the last half a century [31].

The general subdivisions of nanotechnology presented here are not hard and fast since the objectives are ultimately the same, the distinctions can be blurred. The branches that appear to be the most promising are phonon-glass-electron crystals (PGECs), thin-films, and quantum dot superlattices (QDSLs).

PGECs refer to a group of materials that are constructed similar to an ordinary crystal. The distinction results from the fact that the lattice framework readily conducts the electrons, while the phonons are restricted by the voids in the crystal. Furthermore, these voids can be filled with so-called rattler atoms that further inhibit phonons by causing them to rattle around [33]. Popular PGECs being investigated include skutterudites, clathrates, and half-Heusler alloys. Each of these PGECs has a specific chemical formula. For instance, a fully filled skutterudite will have the basic form $\mathrm{ReTm}_{4} \mathrm{Pn}_{12}$ where $\mathrm{Re}$ is a rare earth metal, Tm is a transition metal and $\mathrm{Pn}$ is either $\mathrm{P}, \mathrm{As}$, or $\mathrm{Sb}$ [34]. Each of the PGECs has many formula permutations, and properties vary significantly with dopant levels much like in semiconductors. This leaves numerous possibilities to be investigated, furthermore complicating the issue.

The concept of phonons rattling around or reducing thermal conductivity is a consistent theme for all the nanotechnologies. Once the scale of the heat transfer path, known as mean free path, approaches the wavelength of the phonons the thermal 
conductivity varies significantly from the electrical conductivity. Heat transfer in materials relies on both electron and phonon transport. With the phonons restricted the thermal conductivity is reduced. The theoretical $Z T$ limit of 6.0 is based on reducing the thermal conductivity to its minimum value by engineering the mean free path to approximate the phonon wavelength [33]. Thin-films are materials that are grown layer by layer usually by molecular beam epitaxy (MBE) [7]. The resulting material thickness is on the order of nanometers resulting in a restriction for phonons. The final product consists of alternating layers of different materials chosen for their individual and combined thermoelectric properties. Some thin-films have been used with heat flow perpendicular to the layers, some with heat flow parallel to the layers [35]. In perpendicular heat flow the phonons are restricted when crossing the material boundaries. In parallel heat flow the phonon propagation is restricted by the width of the heat transfer path.

Besides the advantage of lowering thermal conductivity by interfering with phonon transport, nanotechnology actually improves electrical conductivity by increasing electronic density of states through confinement. It suffices here to say that these quantum effects increase the materials electron transport capabilities enhancing its $Z T$. Quantum wells are confined in one dimension (such as in a thin film), nanowires are confined in two dimensions, and quantum dots are confined in three dimensions [36]. QDSLs use the placement of quantum dots to maximize the electronic enhancement and, also, to further intensify the effect of phonon resistance. This phonon effect approaches the theoretical maximum when the quantum dot spacing approaches the phonon wavelength [37]. Although the QDSL seems to maximize all transport effects, the 
production through MBE is extremely slow and will inhibit mass production.

Superlattices made with nanowires can be produced much faster and may offer an acceptable compromise [38].

Still other material investigations are underway including the exploitation of diamond films [39], pressure tuning [35], thermionic emitters and vacuum thermoelements [33]. Some efforts have concentrated on improving the heat transfer characteristics of the contacts and heat sinks to improve the overall COP. For instance, TIMs made from diamond films and carbon nanotubes have been proposed [40]. Other approaches include optimization and finding alternatives to thermoelectrics.

Optimization refers to an approach to maximize performance by using a specific TEC for a specific application [10]. By understanding the exact operating conditions, an ideally rated TEC can be used. Optimization is difficult to apply over a range of operating conditions, because single TECs have a limited range of applicability. The optimization effort has been extended to multiple TECs used in stages, also referred to as cascading. Cascading does produce a broader band of operation and some improvement in $C O P$ [13]. Segmenting is a further extension of optimization. Segmenting uses multiple semiconducting materials inside of the TEC, so that each material operates under its own optimal operating condition [41].

In order to overcome the efficiency problem in TECs, some research efforts are exploring alternative refrigeration techniques. These techniques include vapor compression (VCR), Stirling, pulse tube, sorption and reverse Brayton refrigeration systems [1]. Although these techniques have great potential, these alternative refrigerators are years away from being available. Working models are crude, inefficient, 
and bulky. For instance, the smallest systems that have been built are several kilograms in size and some types of systems have not yet been built at this scale [1]. Recently, a VCR system was built and tested and provided a COP of 1.2 [42]. The primary drawback for theses types of alternate systems is that they are mechanical in nature and have a very low reliability compared to TECs.

TECs inherently have many advantages over the alternate types of refrigeration. TECs are solid-state, so they have no moving parts. Because they have no moving parts, their reliability greatly exceeds any other refrigeration technology currently being considered [1]. Also, TECs are compact and quiet. TECs have no operating fluid or gas that can leak and damage the electronics or the environment. Of all the prospects to remove heat from computer chips, TECs are the only refrigeration technology currently in production. Plus, TECs are compact, lightweight, and relatively inexpensive. TECs appear to be the most desirable cooling alternative; however, TECs still have the efficiency problem that reduces their applicability.

Despite all the recent efforts to improve the performance of TECs, the theoretical prospects have yet to be realized in the marketplace. The efficiencies of TECs currently available on the market are approximately one-third of those on a standard home refrigerator [34]. In order to accommodate the heat generation projected for processors over the next several years, TECs must increase their COPs three-fold [31]. Due to the continuous reduction in size and increases in speed of computer chips, this technology is rapidly outpacing the technology to remove the heat. Current processor designs must be throttled in order to ensure adequate heat removal. According to the International Technology Roadmap for Semiconductors, the typical desktop processor will produce 
$109 \mathrm{~W}$ by 2008 and power will be limited by system level cooling [16]. Figure 1 shows the ITRS prediction of heat removal needs over the next decade. The ITRS predictions are based on Moore's Law, which states that the number of transistors in a computer chip will double about every two years. This indicator has been accurate over the past forty years. Based on these predictions, the need for an improved method of cooling computer chips is critical.

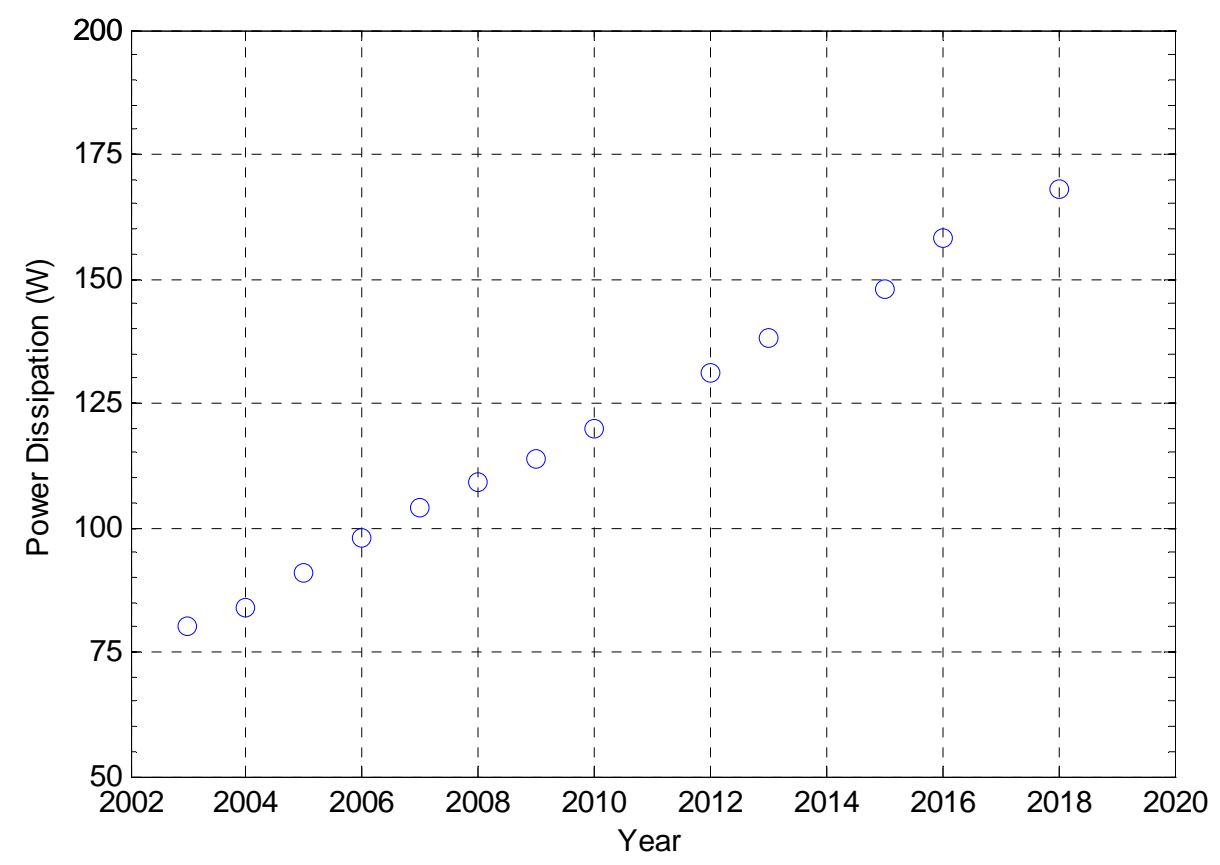

Figure 1. The ITRS prediction for power dissipation requirements of cost-performance, desktop computers over the next several years.

Although TECs have been used to cool device electronics, they have not been widely used to cool computer chips [28]. Computer chips do not heat uniformly, but instead generate hot spots based on the region of the chip that is performing the operations [21]. It makes intuitive sense that a cooling system would be more efficient if it provides greater cooling to the regions that are producing greater heat. The current 
state of the art TEC contains upwards of 30 individual thermocouples connected thermally in parallel, but electrically in series. When the TEC is powered, the cooling is provided uniformly over the entire chip, whether or not it is needed in some parts of the chip. Unnecessarily powered thermocouples produce additional heat to be removed and limit overall cooler performance.

Distributed control is a method that could be implemented to overcome this loss of efficiency. Since a TEC consists of many couples, applying power to each couple discriminately rather than to the entire TEC would allow a greater degree of control that may lead to enhanced performance. The concept of distributed control allows a system that consists of many autonomous, or semi-autonomous, localized controllers called nodes to act on a single system in order to achieve a global control objective [22, 23]. Based on feedback of system response, the individual couples can be controlled to maximize system efficiency. Some couples may be fully powered, some may not be powered, and some may only be partially powered depending on the distribution of heat load in the chip.

\section{Objective}

The objective of this work is to demonstrate viability of a novel approach to improve the performance of TECs. By applying the concept of distributed control to models of simplified TECs, the prospects for this approach may be more accurately gauged. This work will look at the ideal case for applying distributed control, and then generate a distributed control law. The results from applying the law will be compared to the ideal case and to the model for a typical modern TEC. 


\section{CHAPTER II}

\section{THEORY AND MODEL}

A typical TEC is composed of many individual thermocouples, also called elements. Each of these thermocouples is composed of two legs, sometimes referred to as pellets. One leg is composed of an n-type and the other a p-type semiconductor.

Figure 2 shows the typical configuration of an element [34].

The n-type and p-type legs are connected electrically in series to complete the circuit. The charge carriers are electrons in the n-type leg and holes in the p-type leg.

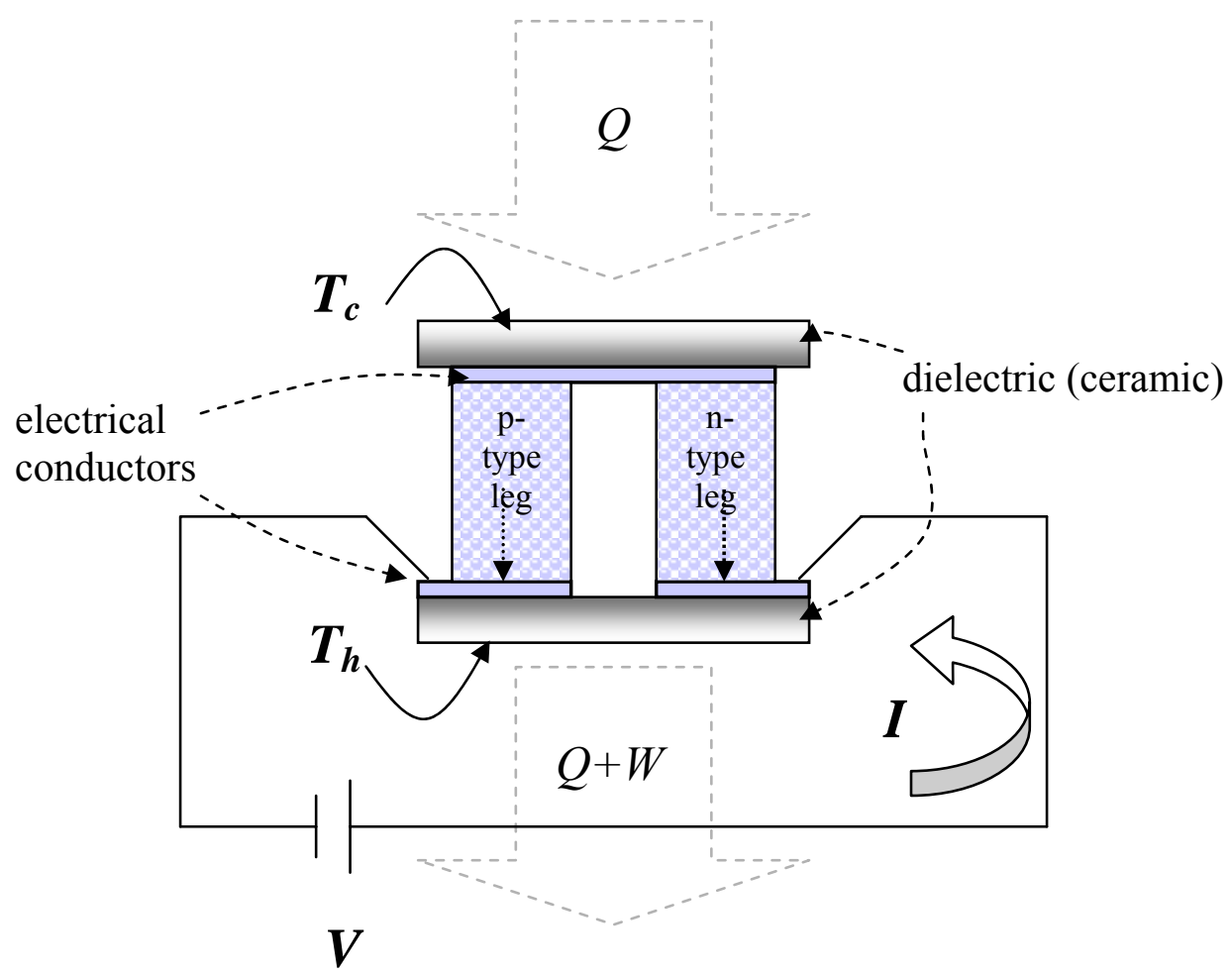

Figure 2. Schematic of a typical thermoelectric cooling element. 
When current flows due to an applied voltage, the charge carriers are forced to one-side depending on the direction of current flow. In Figure 2, the charge carriers will be forced down to the side denoted by $T_{h}$. This results in a "hot side" because the charge carriers are carrying heat from the cold side. This temperature gradient results in heat being absorbed on the cold side, $Q$. The heat and the work applied to the thermocouple, $Q+W$, must be removed from the hot side.

The TEC consists of many individual thermocouples connected electrically in series, but thermally in parallel. Figure 3 shows a drawing of a typical TEC with 12 couples and a transparent top piece. When a voltage is applied to the cooler, all thermocouples are powered simultaneously. The heat is absorbed on the cold side and released on the hot side. Ceramic plates are used to provide a uniform heat transfer surface. Figure 4 is a photograph of an actual modern TEC with the individual legs visible between the ceramic plates.

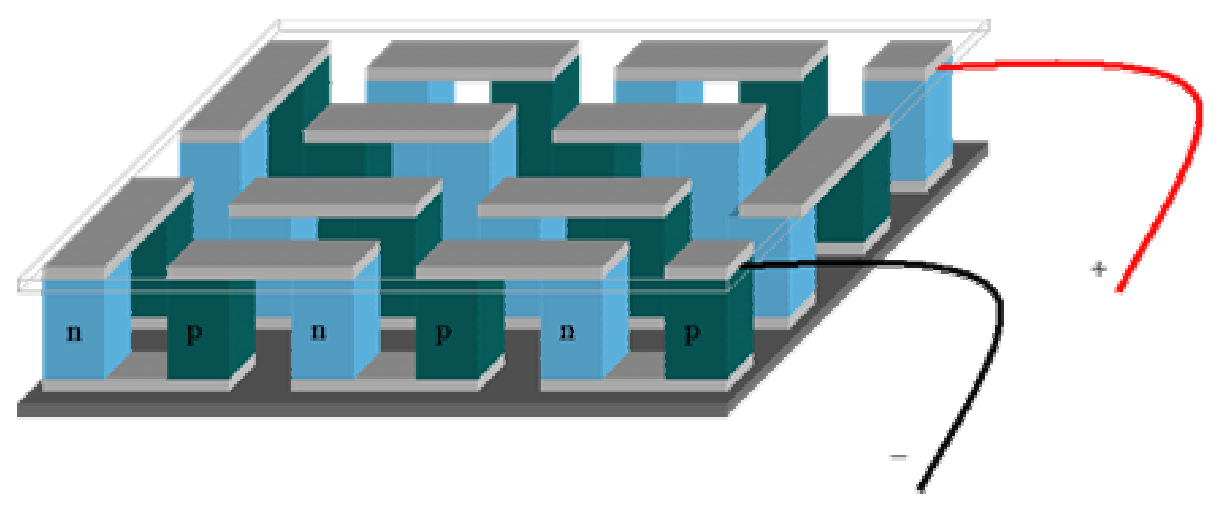

Figure 3. Drawing of a typical TEC with 12 couples and a transparent top piece. 


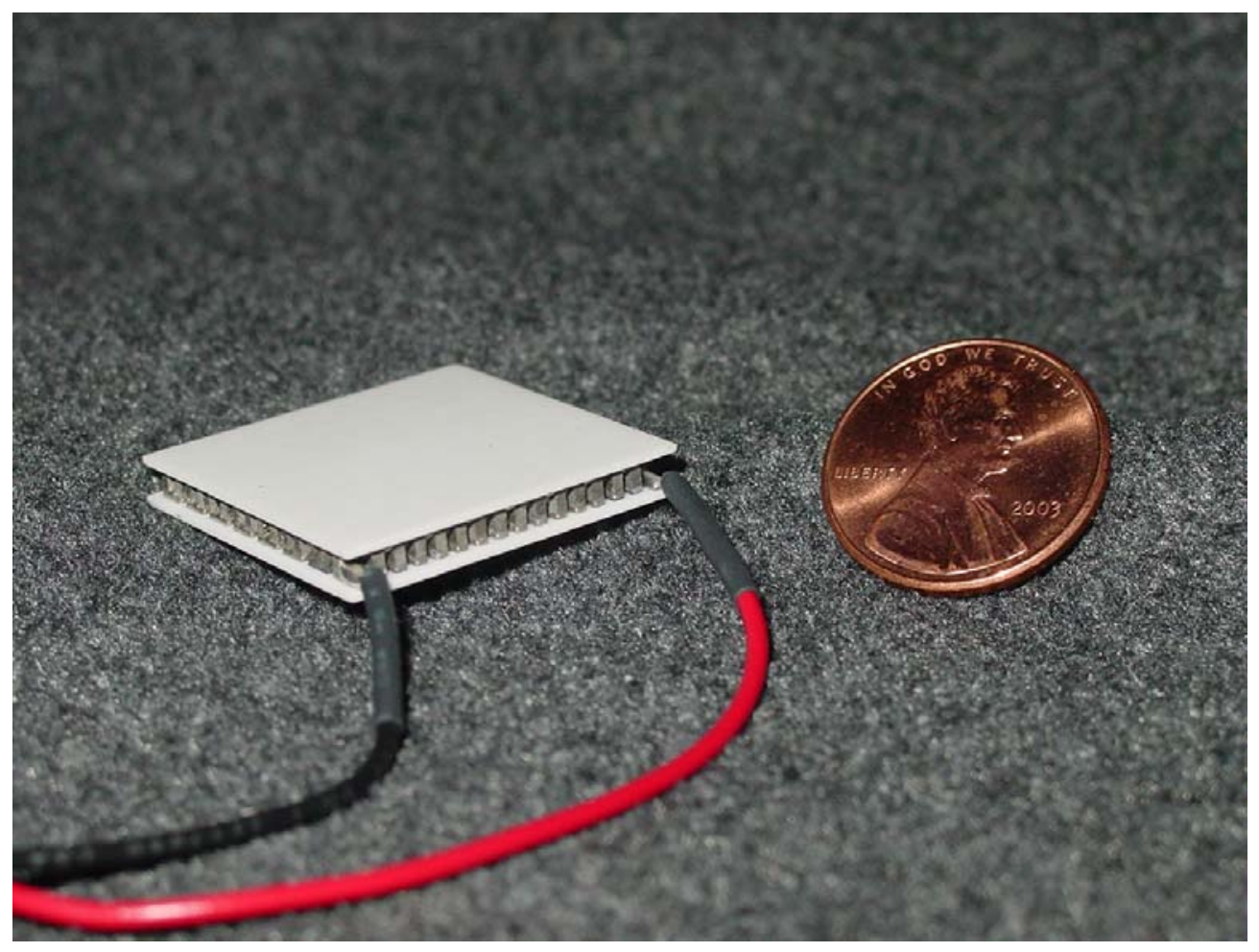

Figure 4. Photograph of a modern TEC with 127 couples shown with a penny for size comparison.

A TEC is usually square and essentially a two-dimensional array of thermocouples. The ceramic plates are in direct contact with the surface of a computer chip. For this analysis, a one-dimensional (linear) array is considered for simplicity. Each area of the computer chip that is in contact with an individual couple is arbitrarily considered to be a separate and distinct heat-producing portion. Using the electrical analogy for heat transfer, the schematic for $n$ number of thermocouples is given in Figure 5. $Q_{\text {geni }}$ represents the heat being produced by the $i^{\text {th }}$ portion of the chip. $Q_{i}$ represents the heat being removed by each thermocouple. $Q_{\text {condi }}$ represents the heat that is transferred by conduction between the $i^{\text {th }}$ and the $i+1^{\text {th }}$ portions of the computer chip. 


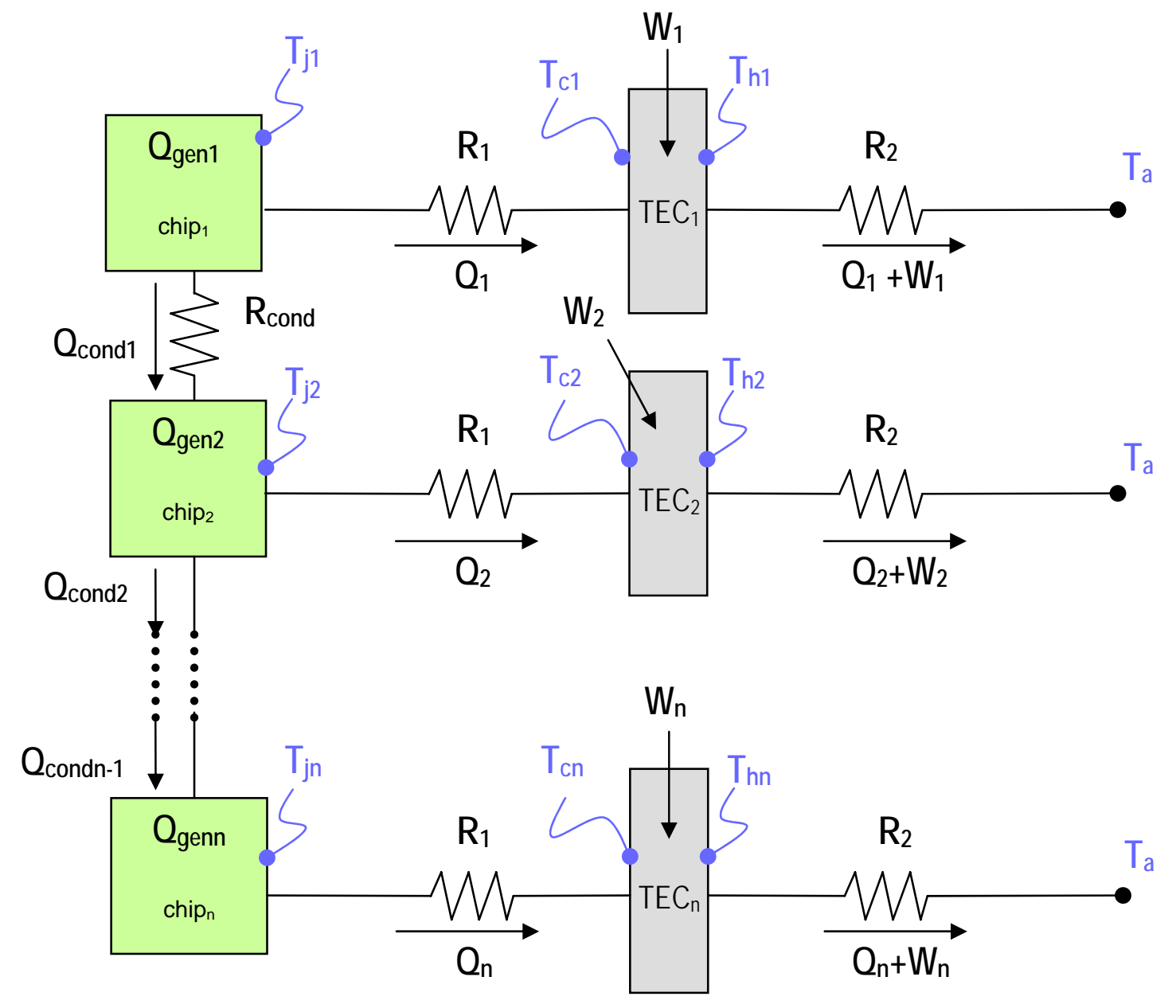

Figure 5. A schematic diagram using the electrical analogy for heat transfer to represent $n$ thermocouples removing heat from an equal number of computer chip portions.

$R_{1}$ and $R_{2}$ represent the thermal resistances between the chip and thermocouple, and between the thermocouple and the ambient environment, respectively. $R_{l}$ is an equivalent resistance that accounts for chip packaging (including a heat spreader if used), thermal interface material (TIM), and contact resistances. $R_{2}$ accounts for TIM, contact resistances, and the effectiveness of the fin-fan system. Likewise, $R_{\text {cond }}$ is an equivalent lateral thermal resistance between portions of the computer chip. $W_{i}$ is the amount of work the thermocouple requires to remove the heat, $Q_{i}$, from the chip. Due to the first 
law of thermodynamics, however, the sum of the work and the heat, $Q_{i}+W_{i}$, must be removed from the system. $T_{j i}$ represents the junction temperature, which is at the surface of the computer chip. $T_{c i}$ and $T_{h i}$ are the temperatures of the cold and hot surface of thermocouple respectively. Finally, $T_{a}$ is the ambient temperature.

The solution is found by solving a system of simultaneous algebraic equations.

The heat removed by each thermocouple, $Q_{i}$, is [27],

$$
Q_{i}=2\left[\alpha I T_{c i}-\frac{1}{2} I_{i}^{2} \frac{\rho}{G}-k G \Delta T_{i}\right] \text {, }
$$

where $\alpha$ is the Seebeck coefficient, $I$ is the electrical current through the device, $T_{c i}$ is the cold-side temperature, and $\Delta T_{i}$ is given by

$$
\Delta T_{i}=T_{h i}-T_{c i} .
$$

$T_{h i}$ is the hot-side temperature and $T_{c i}$ is the cold-side temperature of the individual thermocouples. Referring back to (6), $G$ is a geometric factor equal to the cross-sectional area divided by the height of a thermocouple, $\rho$ is the electrical resistivity, and $k$ is the thermal conductivity. The factor of two is required because each couple is composed of two pellets. The first term in brackets represents the heat being pumped by the couple due to the Peltier effect. This benefit is offset by the Joule heating in the device (the second term in brackets), and the parasitic heat flow in the couple due to the temperature gradient (the last term in brackets). Parasitic heat flow is the term given to the Fourier heat transfer that occurs in the direction opposite the desired direction due to an adverse temperature gradient. The analysis parameters used here are based on a specific Melcor $^{\mathrm{TM}}$ device, Model CP5-31-06L and are given in Table 1. It should be noted that the contact resistances, $R_{1}$ and $R_{2}$, vary widely with temperature and surface preparation. A constant value is used here for simplicity and scaled to size accordingly [28]. 
Table 1. Analysis Parameters.

\begin{tabular}{|l||l|}
\hline Parameter & Value \\
\hline \hline geometric factor, $G$ & $1.196 \mathrm{~cm}$ \\
electrical resistivity, $\rho$ & $1 \times 10^{-5} \Omega \mathrm{m}$ \\
thermal conductivity, $k$ & $1.5 \mathrm{~W} / \mathrm{mK}$ \\
lateral conductivity, $k_{\text {cond }}$ & $10.0 \mathrm{~W} / \mathrm{mK}$ \\
Seebeck coefficient, $\alpha$ & $\pm 2 \times 10^{-4} \mathrm{~V} / \mathrm{K}$ \\
thermal resistance, $R_{l}$ and $R_{2}$ & $10 \mathrm{~K} / \mathrm{W}$ \\
lateral resistance, $R_{\text {cond }}$ & $20 \mathrm{~K} / \mathrm{W}$ \\
ambient temperature, $T_{a}$ & $25^{\circ} \mathrm{C}$ \\
\hline
\end{tabular}

The work done by each thermocouple, $W_{i}$, is given by

$$
W_{i}=2\left[I_{i}{ }^{2} \frac{\rho}{G}+\alpha I_{i} \Delta T_{i}\right]
$$

[28], and the terms are analogous to those previously discussed regarding (6).

The remaining equations used to define the system are obtained by balancing heat into and out of the chip sections and each thermocouple, where the heat is conserved for each portion of the chip as in

$$
Q_{\text {geni }}=Q_{\text {condi }}-Q_{\text {condi }-1}+Q_{i} .
$$

The heat transferred between two portions of the chip is given by

$$
Q_{\text {condi }}=\frac{T_{j i}-T_{j i+1}}{R_{\text {cond }}} .
$$

The heat removed by a thermocouple is equal to (6) and is also given by

$$
Q_{i}=\frac{T_{j i}-T_{c i}}{R_{1}} .
$$


The heat and work removed by the system is given by

$$
Q_{i}+W_{i}=\frac{T_{h i}-T_{a}}{R_{2}}
$$

The algebraic system is nonlinear with respect to current, so it must be solved iteratively for temperatures, heat loads, and currents. MATLAB ${ }^{\mathrm{TM}}$ code was written to solve a system of simultaneous algebraic equations using matrix computations. The equation used to solve the matrix is given by

$$
b=A^{-1} c .
$$

The term $b$ is the solution matrix and is given by

$$
b=\left\langle\begin{array}{lllllll}
T_{j i} & Q_{i} & T_{h i} & T_{c i} & \Delta T_{i} & W_{i} & Q_{\text {condi }}
\end{array}\right\rangle
$$

The term $\mathrm{A}$ is the coefficient matrix and is given by

$$
A=\left(\begin{array}{ccccccc}
0 & 1 & 0 & -2 \alpha I_{1} & 2 k G & 0 & 0 \\
0 & 0 & 0 & 0 & -\alpha I_{1} & 1 & 0 \\
-1 & R_{1} & 1 & 0 & 0 & 0 & 0 \\
0 & 0 & -1 & 1 & 1 & 0 & 0 \\
0 & R_{2} & -1 & 0 & 0 & R_{2} & 0 \\
0 & 1 & 0 & 0 & 0 & 1 & 0 \\
-1 & 0 & 0 & 0 & 0 & 0 & R_{\text {cond }}
\end{array}\right)
$$

The term $c$ is the input matrix and contains all the $I$ terms that are nonlinear and the heat generation terms and is given by

$$
c=\left\langle-I^{2} \rho / G \quad 2 I^{2} \rho / G \quad 0 \quad 0 \quad-T_{a} \quad Q_{\text {geni }} \quad 0\right\rangle .
$$

The code accepts heat loads, $Q_{g e n}$, as an array of length $n$, and then applies the values to $c$. The code then adjusts the current levels to each thermocouple and performs the calculations to determine the resulting temperatures and COPs. The optimization function fmincon, provided in $\mathrm{MATLAB}^{\mathrm{TM}}$, is used to maximize COP for each heat 
distribution scenario. By maximizing COP, the optimum performance of the TEC is achieved for the given heat load. Note that the effective $C O P$ of the entire device as determined for the distributed control configuration will differ from (1) for a typical serial TEC and is given by

$$
C O P=\frac{\sum_{i=1}^{n} Q_{\text {gen } i}}{\sum_{i=1}^{n} W i} .
$$

This equation gives some insight to the inherent advantage of distributed control. If a couple is not powered, it will still provide some heat removal due to Fourier heat conduction. This advantage is termed "free" heat removal and can result in scenarios that make comparison complicated. For instance, in some operating regimes the resulting COP will be infinite or negative and must be interpreted cautiously. The analysis in this work will concentrate on the regimes where $C O P$ is both positive and finite.

Because of the "free" heat removal, distributed control is anticipated to improve $C O P$ as much as three times that of a serial type TEC for nonuniform heat loads. This amount of improvement is significant and should be sufficient for more widespread application of TECs. Distributed control could provide the improvement in $C O P$ required to cool the next generation of desktop computer processors. 


\section{CHAPTER III}

\section{RESULTS}

\section{Two-Couple Case}

As previously stated, a typical TEC can consist of many thermoelectric elements. Initially, let us consider a simple case in which the chip is divided into only two portions. One portion represents the area of a chip that is performing calculations and is, therefore, producing heat, $Q_{g e n}$. The other portion represents an idle portion of the chip and is not generating any heat. However, heat, $Q_{c o n d}$, will be conducted laterally to the idle portion

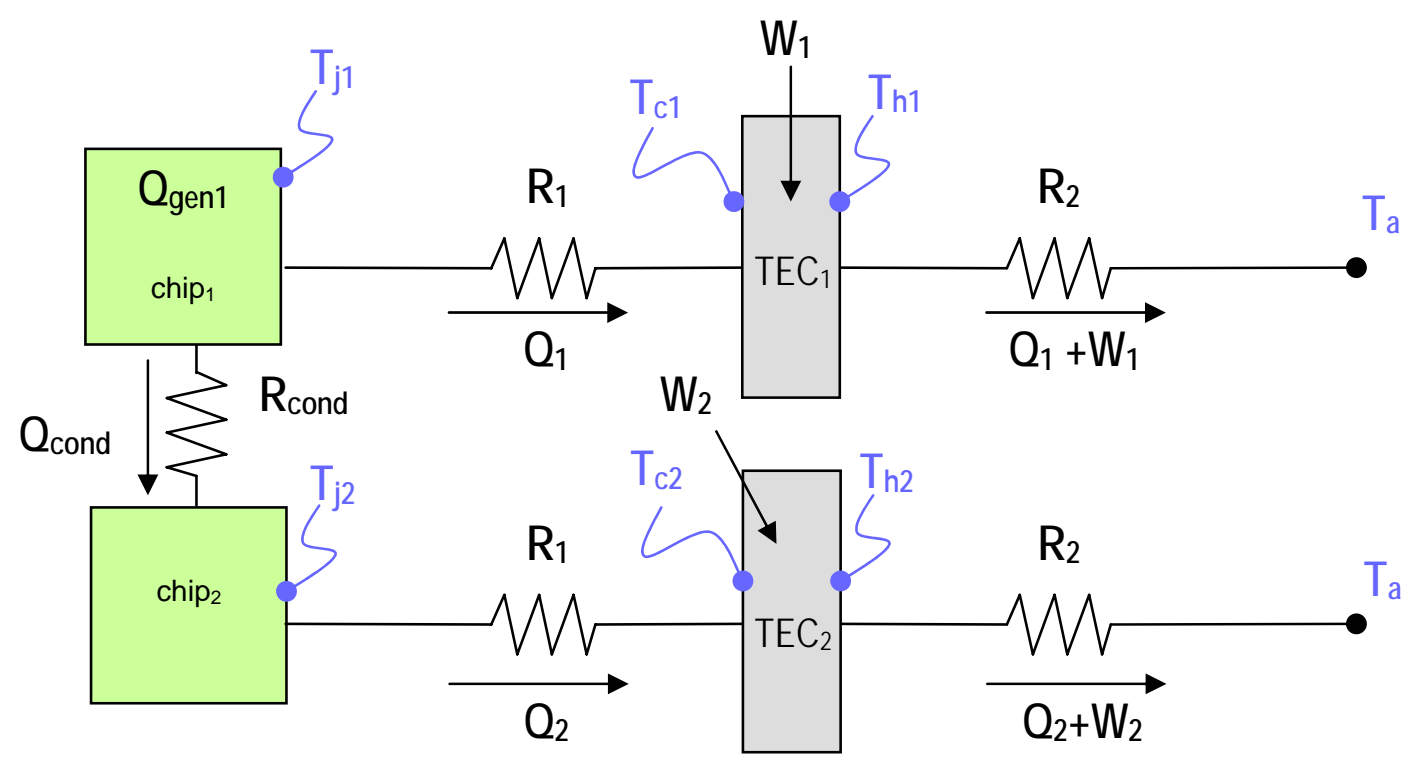

Figure 6. A schematic diagram representing two thermocouples removing heat from two portions of a computer chip.

from the operating side. Each portion of the chip is in thermal contact with an individual thermocouple of a TEC. Thus, this TEC has only two thermocouples. This simple case is illustrated in Figure 6, which is an abbreviated version of Figure 5. 
In this case, the thermocouples will be independently powered, as if connected electrically in parallel to simulate distributed control. The heat removed by the powered thermocouple is $Q_{1}$. Heat, $Q_{2}$, will be transferred from the idle portion of the chip to the idle TEC by conduction and removed to ambient conditions. In a real functioning system, couples would not normally be powered until a portion of the chip reaches a predetermined threshold temperature that could result in degraded chip performance. When the threshold condition is met, the appropriate thermocouples would be powered to cool the chip within acceptable limits. Computer chip performance is reported to degrade

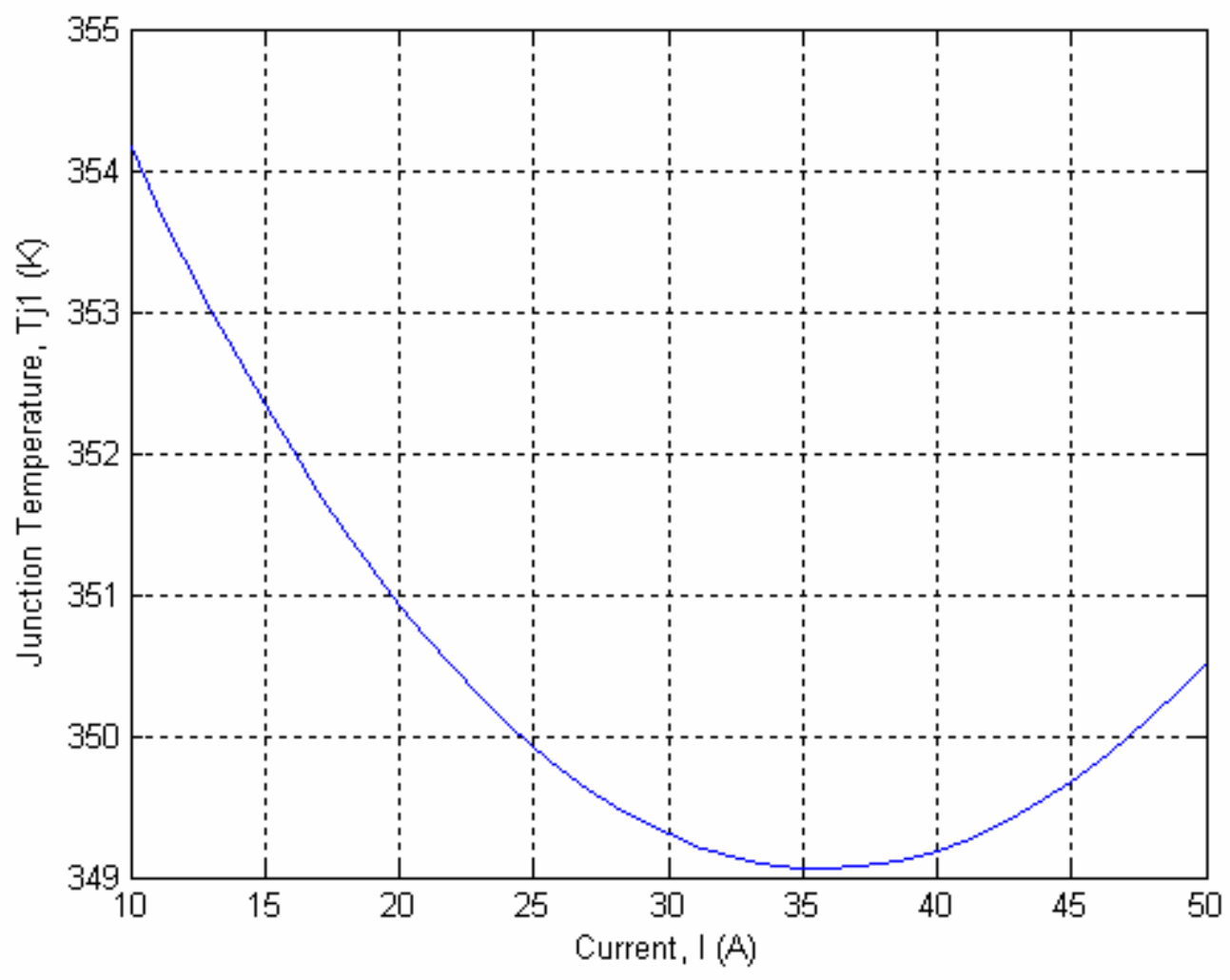

Figure 7. Junction temperature vs. current for a constant heat load.

for a junction temperature of $85^{\circ} \mathrm{C}$, so this temperature will be used for the threshold, $T_{\text {jmax }}[1]$. Whenever the powered portion of the chip in the present example is producing 
$2.9 \mathrm{~W}$ or less, $T_{j l}$ never reaches the threshold criterion. The heat is adequately transferred without the aid of the TEC. Once the chip produces greater than $2.9 \mathrm{~W}, T_{j 1}$ will rise above $T_{\text {jmax }}$ and the TEC must be powered to prevent the chip from operating in a forbidden mode. Figure 7 shows the effects on $T_{j l}$ when the TEC is powered and $Q_{g e n l}$ is $3.0 \mathrm{~W}$. For the constant heat load, as the current is increased, $T_{j l}$ decreases until it reaches a minimum, and then increases again as Joule heating inside the TEC overcomes the cooling provided. Figure 8 shows the corresponding $C O P$ for the system as a function of current. At lower current levels, the $C O P$ is negative and varies significantly.

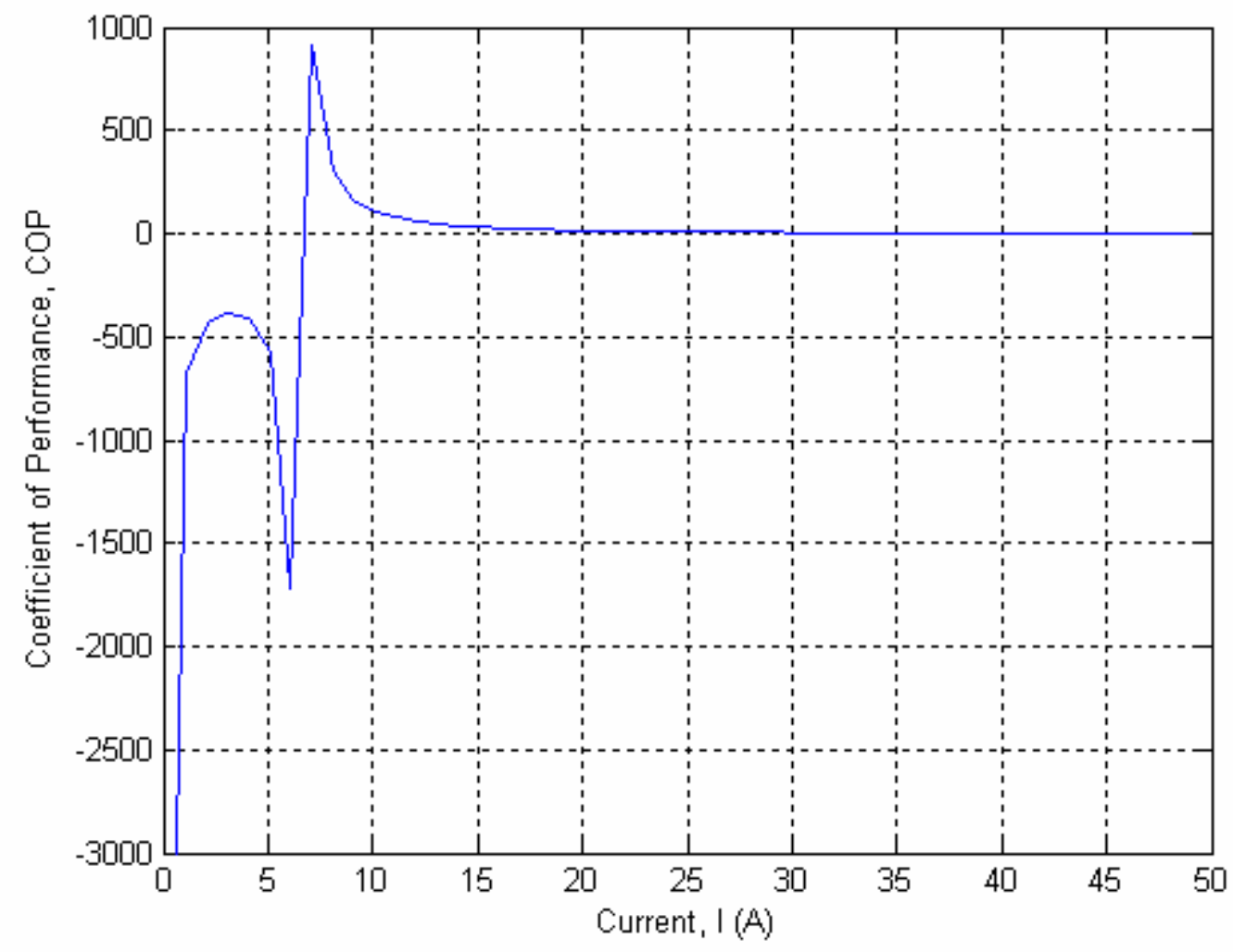

Figure 8. Coefficient of performance vs. current for a constant heat load. 


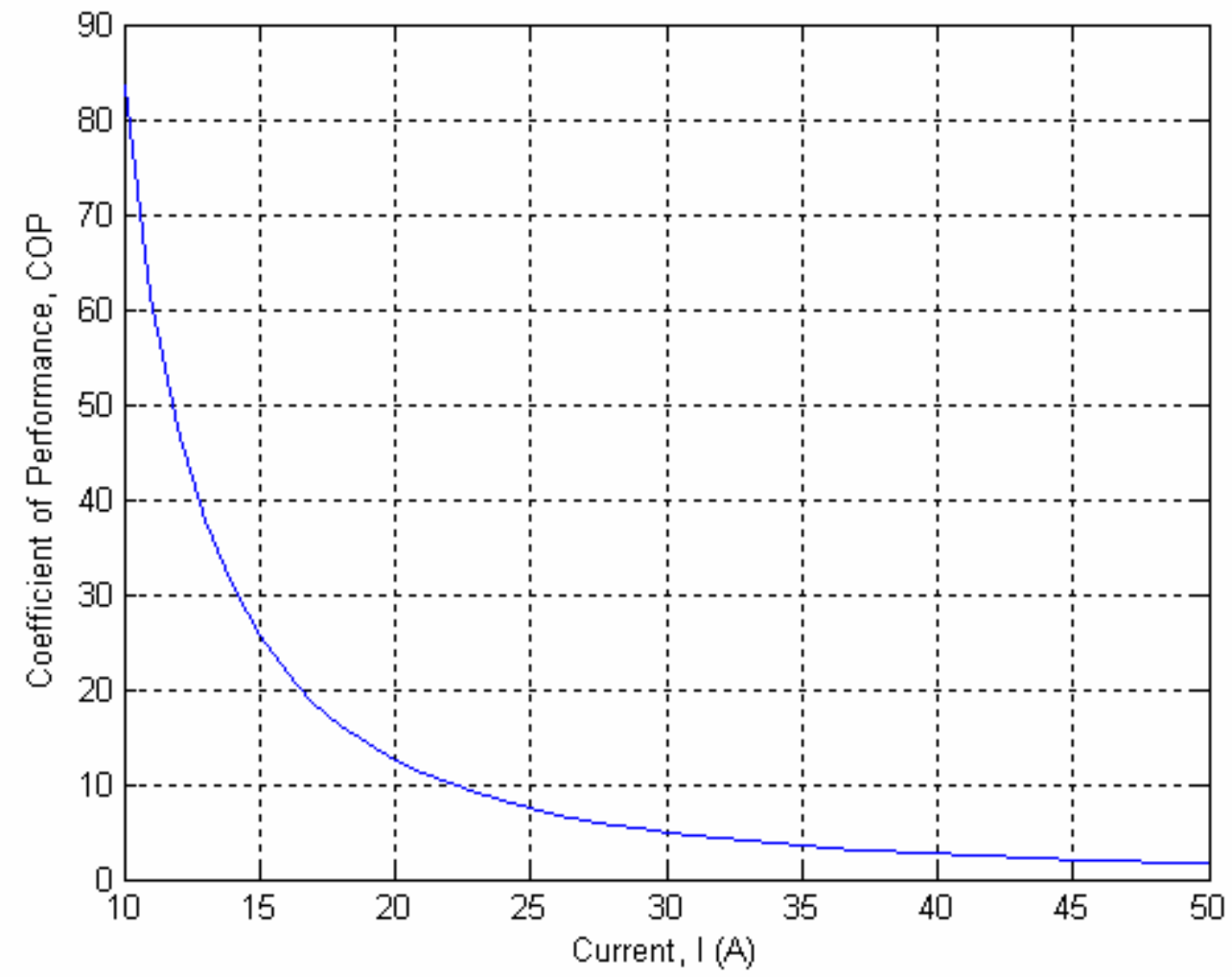

Figure 9. Coefficient of performance vs. current for a constant heat load (cropped).

This behavior is expected due to multiple modes of operation provided by a TEC. The TEC functions as a generator at these lower current levels resulting in a negative work term. This erratic behavior of $C O P$ at low current levels makes comparison difficult and the information gained speculative. Because of this behavior, the remaining figures will only represent the operating regions where $C O P$ is positive and finite. Figure 9 is a cropped version of Figure 8 that illustrates the operating region that will be considered in the remainder of this work.

The focus of this work is to examine the possibility of improving the efficiency of the cooler by controlling small sections of the entire cooler independently. Two configurations are used to demonstrate this feature. In both cases, a simulated chip is 
heated nonuniformly and cooled by two thermocouples. For the baseline case, the two couples are synchronized such that the current through each is the same. This is how typical, modern TECs are arranged. When $T_{j l}$ reaches $85^{\circ} \mathrm{C}(358 \mathrm{~K})$, both couples are powered simultaneously as a single unit. The current provided to each couple is the same, and the minimum amount of current required to maintain $T_{j l}$ below the threshold temperature, $T_{j \max }$, is applied. In the second case, the thermocouples are operated independently with different currents applied. The first couple is powered until it can no longer maintain the threshold criterion for the increasing heat load. The second couple is then powered as required to assist in heat removal.

Figure 10 shows the relationship of the currents applied in both cases for heat

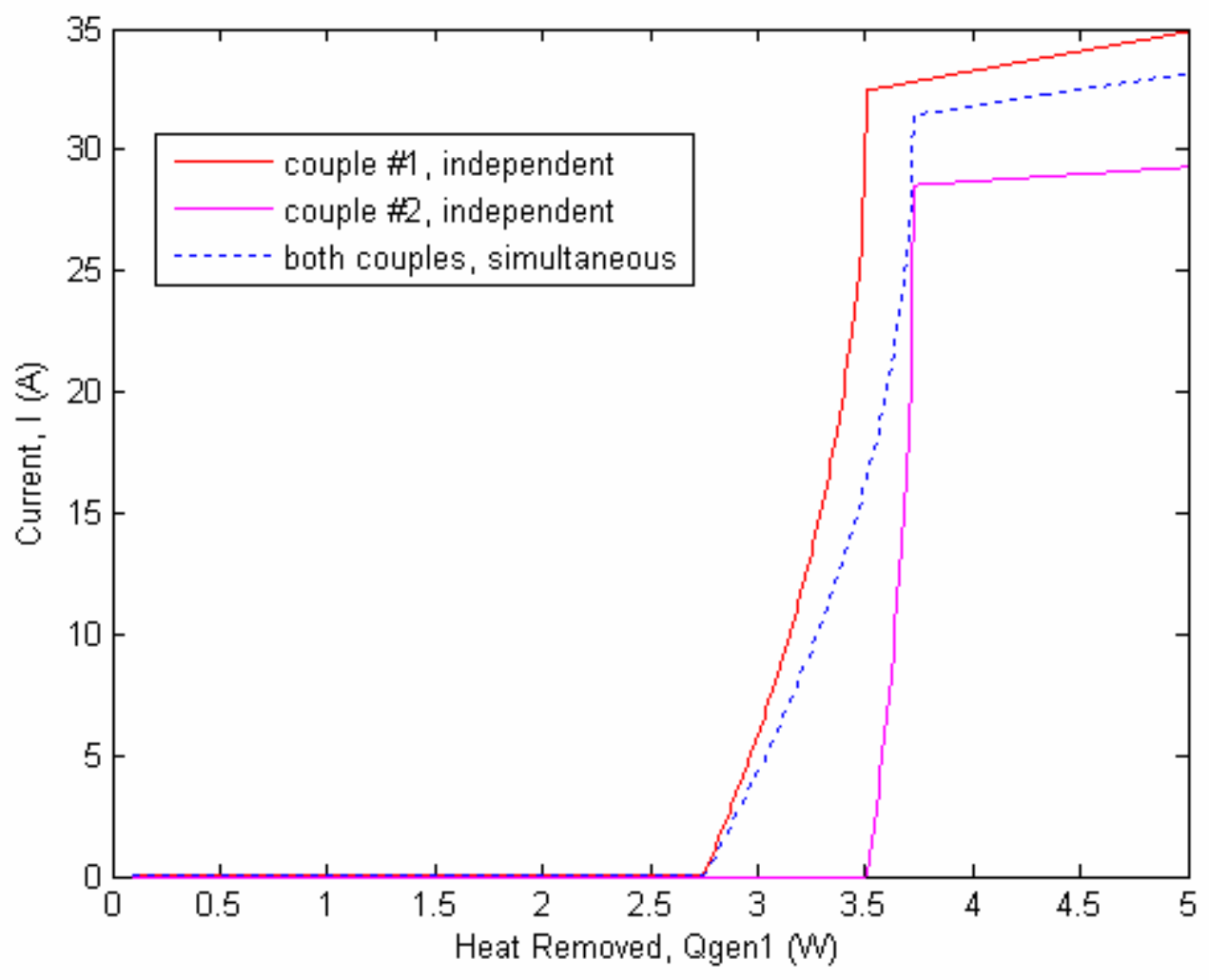

Figure 10. The current required for various heat loads, $Q_{\text {gen } 1}$. The solid curves represent the currents to each couple when they are activated independently. The dashed curve is the current through both couples activated simultaneously. 
loads ranging from 0 to 5.0 watts. The single unit and the independent couples must both be powered when the heat can no longer be removed by Fourier heat transfer alone. This occurs near 2.9 watts. The single unit requires less current to remove the increasing heat load because both couples are powered. The first independent couple can no longer remove enough heat to maintain $T_{j 1}$ below the threshold criterion at about 3.5 watts. Therefore, the second couple is powered. At a slightly higher heat load, neither case can maintain the threshold criterion as the currents increase without bound.

In Figure 11, the temperatures are plotted for each couple and each case versus

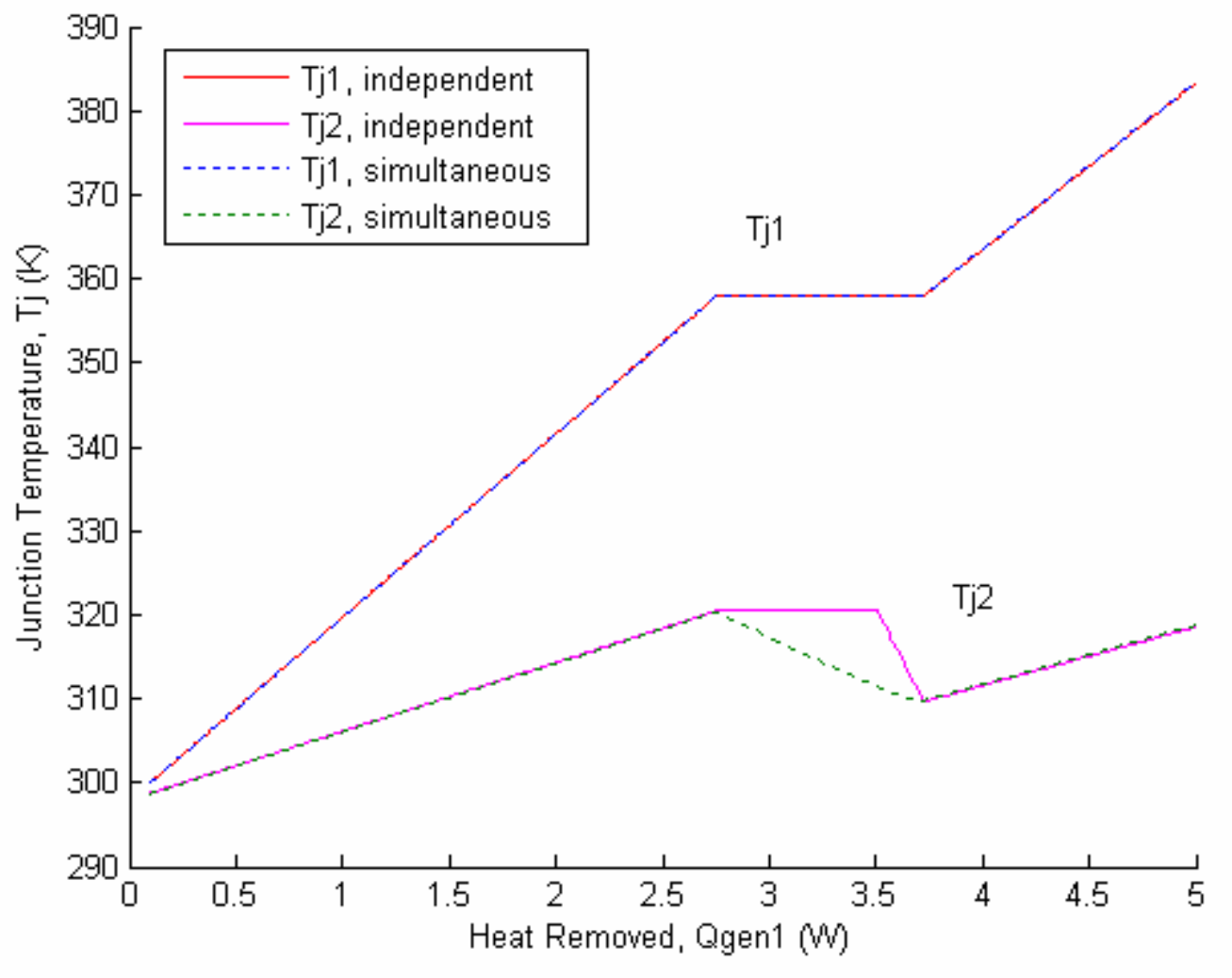

Figure 11. The junction temperatures for various heat loads, $Q_{\text {gen } 1}$. The solid curves represent the currents to each couple when they are activated independently. The dashed curve is the current through both couples activated simultaneously. 
increasing heat load. The plots are virtually identical except in a small region where $T_{j 2}$ for the two cases diverges. In the case of a single unit, $T_{j 2}$ is unnecessarily lowered by the portion of the cooler that is not experiencing heating. This reduction occurs at the instant the unit meets the threshold criterion and the unit is powered. The second couple is powered unnecessarily. This is illustrated in Figure 11 where the two plots for the second couple diverge at around 2.9 watts. This exemplifies the benefit of distributed control. Although this is only a small portion of the operating range, this case only has two thermocouples. The benefit should be more pronounced as more thermocouples are considered.

In the case of the independent couples, the reduction in $T_{j 2}$ does not occur until a much higher heat load. At heat loads greater than about 3.6 watts, neither case can maintain $T_{j l}$ below the threshold criterion. Interestingly, the independently controlled couples maintain the entire chip at a slightly more uniform temperature throughout the entire operating range. This feature is significant and should result in increased chip lifetime by reducing internal thermal stresses. Chip lifetime and reliability is a major consideration of thermal management.

In Figure 12, the COPs are plotted versus heat load for each case. For the independent case, the $C O P$ has a discontinuity around 3.5 watts caused by the second couple being powered. Both cases level out at a little higher than 3.7 watts. The analysis demonstrates that for the present conditions independent couples do not have a better overall thermal efficiency. However, this unexpected result can be attributed to the degree of lateral conduction in the chip. Consider, for example, when the resistance to lateral conduction approaches infinity, the lateral conduction approaches zero. Each 


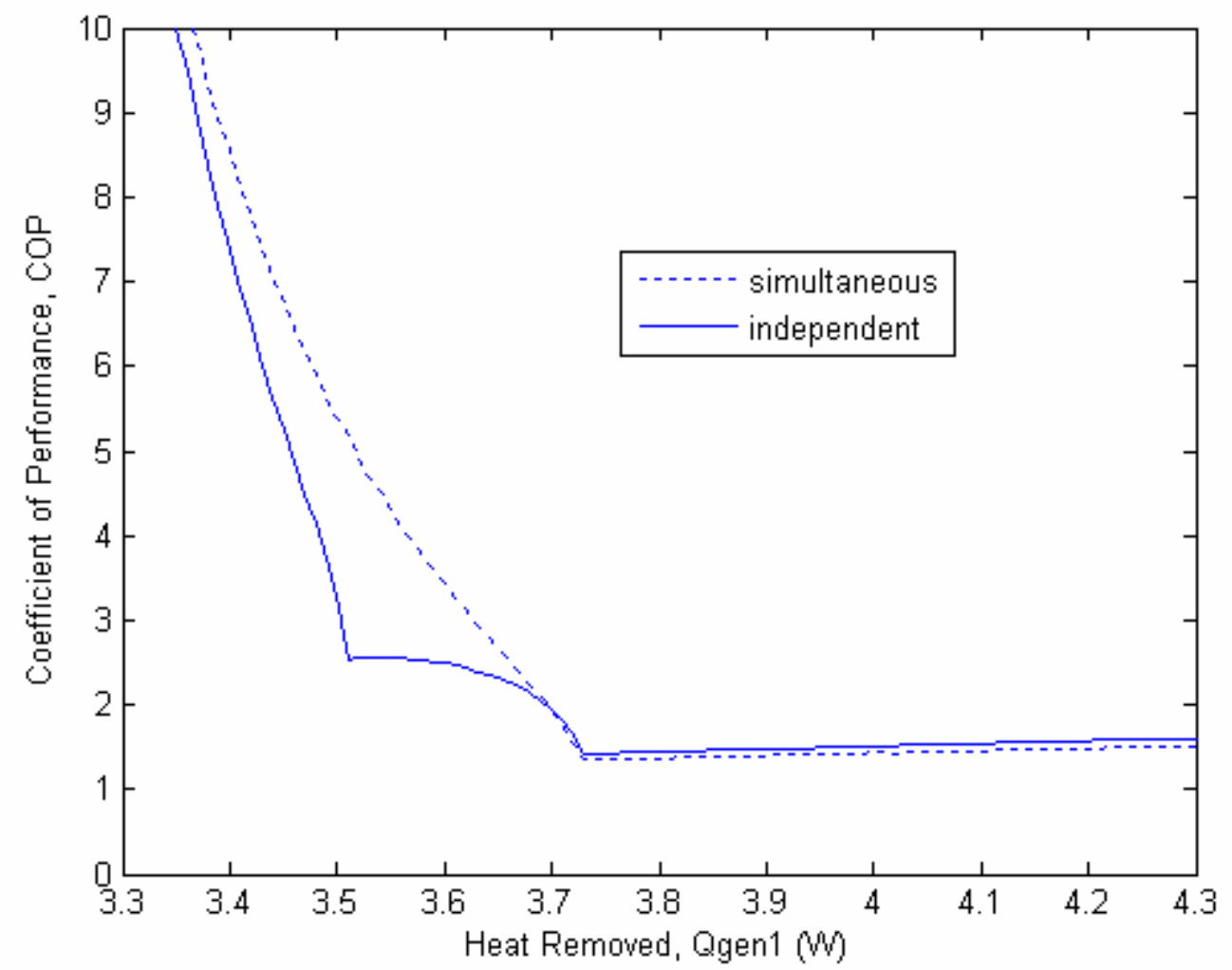

Figure 12. COP for various heat loads, $Q_{g e n}$. The solid curve represents the two couples operating independently, and the dashed curve is when they are operating simultaneously (high lateral conductivity).

thermocouple would be solely responsible for the heat production of its corresponding chip portion. If one portion of the chip produced heat and the other was idle, then the heat distribution would be discontinuous and nonuniform. This scenario represents the ideal case for distributed control.

Next, consider the opposite case in which lateral conduction approaches infinity. This corresponds to the case in which the heat produced is distributed uniformly across the entire chip. The heat load would be the same for all thermocouples. In this case, distributed control offers no advantage. This case can be approximated by the use of a 


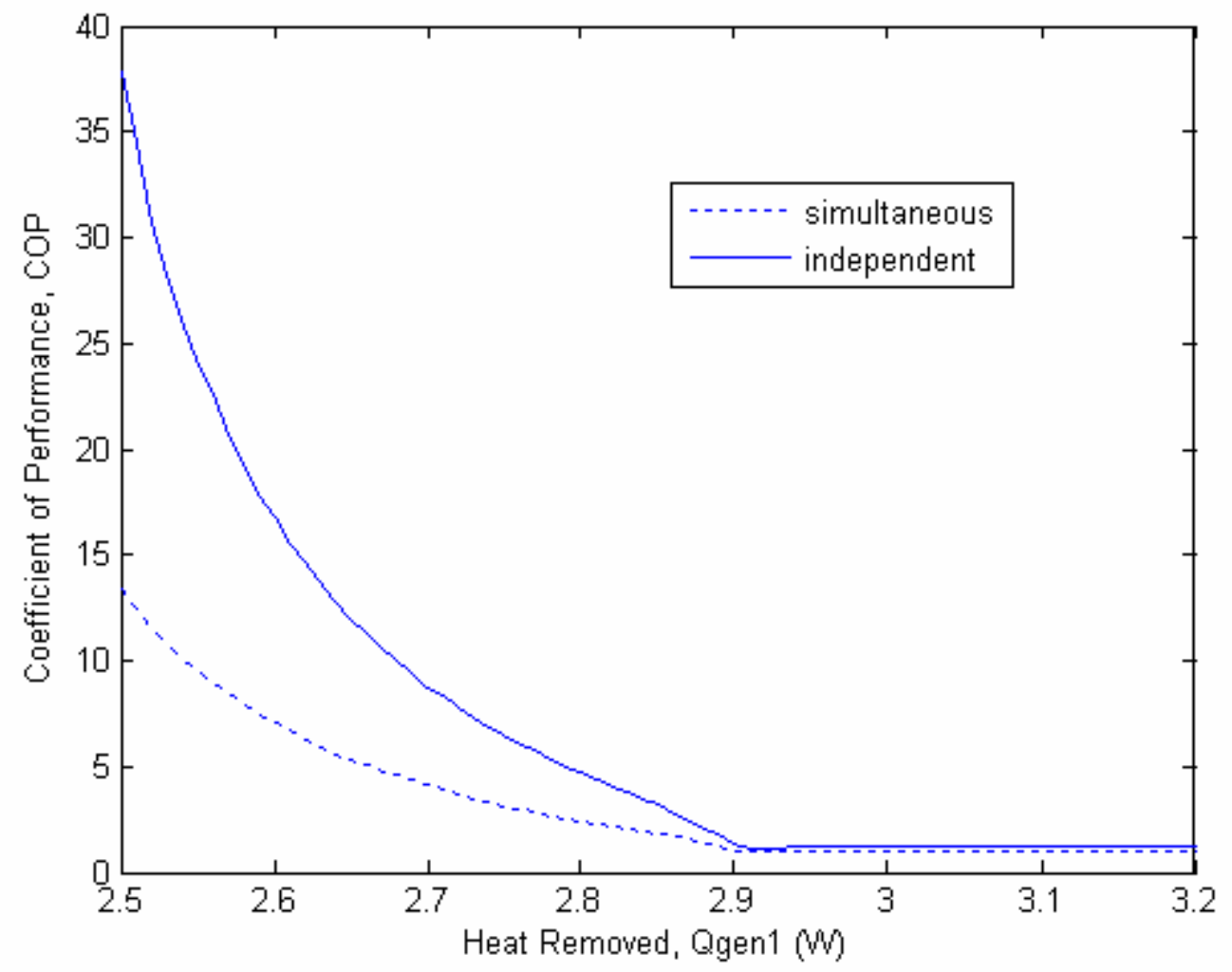

Figure 13. $C O P$ for various heat loads, $Q_{\text {genl }}$. The solid curve represents the two couples operating independently, and the dashed curve is when they are operating simultaneously (low lateral conductivity).

heat spreader. A chip using no heat spreader would more accurately simulate the low conductivity case. By changing the lateral thermal conductivity used in the model to more closely represent the case in which no heat spreader is used, results are changed significantly. In fact, the efficiency of the independent configuration is highly sensitive to the lateral conduction. Figure 13 shows the $C O P$ for a chip whose lateral resistance is an order of magnitude larger than in Figure 12. The efficiency of the independently controlled device is significantly larger than that of the single unit. 


\section{Five-Couple Case}

Now that the benefits of the two-couple case have been identified and examined, the model can be extended to a more complicated scenario. The next case will examine an array of five thermocouples corresponding to a discretized chip with five nodes. For simplicity and ease of comparison, again, only the first portion of the chip corresponding to the first thermocouple will produce heat; this is the worst-case scenario for a TEC that is cooling uniformly. For this example the input values of heat being generated by the first portion of the chip, $Q_{g e n}$, range from $0.0 \mathrm{~W}$ to $5.0 \mathrm{~W}$. Again, $85{ }^{\circ} \mathrm{C}$ will used for $T_{\text {jmax }}$. At heat loads greater than $4.8 \mathrm{~W}$, the five-thermocouple configuration is unable to maintain $T_{j 1}$ less than $T_{j m a x}$. The resulting computed values are not considered in the analysis since the system would be operating in failure mode.

The optimal values of $C O P$ are plotted in Figure 14 as a function of heat load. For comparison the values of $C O P$ for the same configuration with the thermocouples connected in series are also plotted. Figure 14 illustrates the maximum theoretical gain in COP from applying distributed control for the five-thermocouple model. The optimum values represent an $80 \%$ increase over the serial values averaged throughout the entire operating range. Note that in some operating regimes, the optimum values are as much as three times the serial values. This is a remarkable gain.

In order to achieve this gain, however, a distributed control rule must be applied to allow the system to respond appropriately via sensors and actuators to approximate the optimum condition. More specifically, the nodes within the system must be able to sense specified local parameters, apply a distributed control rule in order to determine appropriate system response, and then apply that response to the system. To determine a 


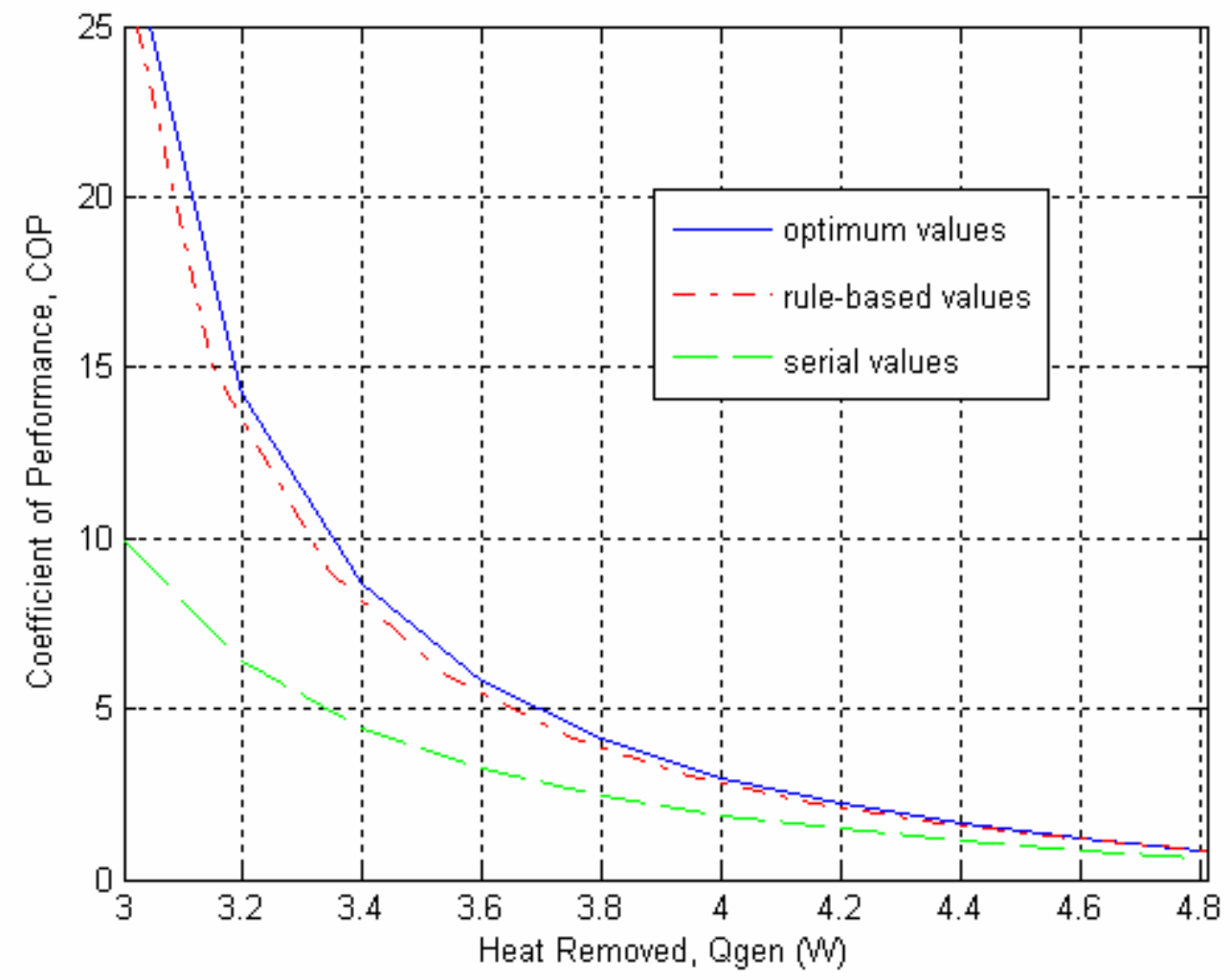

Figure 14. Plots of the optimum $C O P$ and the $C O P$ calculated from the distributed control rule versus the heat removed from the chip. Also shown are the comparable values of $C O P$ for a typical TEC in which the couples are powered in series.

distributed control rule, the parameters that may be sensed by the system must be considered. Ideally, the amount of cooling should be directly related to the amount of heating. The most reasonable way to sense the amount of heat generation is by measuring the resulting temperatures. The local temperatures that are available to be directly sensed by the TEC are the hot-side and cold-side temperatures, $T_{c i}$ and $T_{h i}$, of the individual couples. If the optimum current (the current that maximizes $C O P$ ) may be determined by its relationship with the sensed parameters, then this relationship is essentially the distributed control rule. 


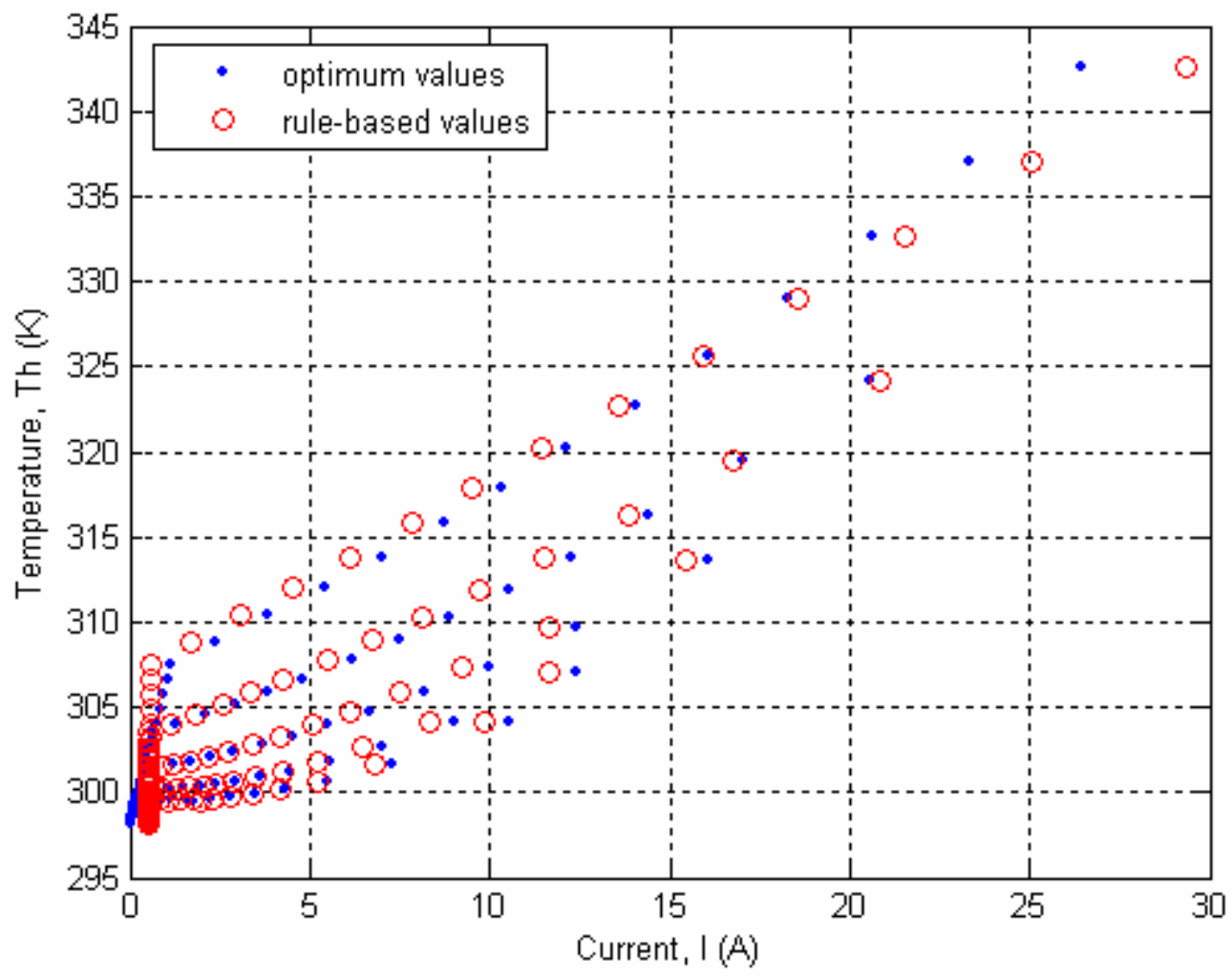

Figure 15. A plot of the optimum $C O P$ data (dots) compared to the data calculated from the distributed control rule (circles) as calculated using multiple linear regression.

In order to find this distributed control rule, $T_{c i}$ and $T_{h i}$ were compared to the optimum current using curve-fitting techniques. Several different methods were attempted including variations of multiple linear and multiple polynomial regression with varying results. The resulting equation of the first method, multiple linear regression, is given by

$$
I_{i}=1.7643+0.7650 \cdot\left(T_{h i}-300\right)-0.1448 \cdot\left(T_{c i}-300\right)
$$

in which $T_{c i}$ and $T_{h i}$ are provided in units of Kelvin and the resulting $I_{i}$ is in watts.

The resulting rule-based values of current from applying (18) are plotted in Figure 15 as circles. The dots in Figure 15 signify the optimum values of current. In this figure, 
each thermocouple has a distinct curve. There are five curves with the couple adjacent to the heat source having the highest temperatures and, therefore, requires the most current. The other couples require less current the farther removed they are from the heat source. The optimum values result in obviously curved plots, so the linear rule-based values are limited in closeness of fit.

Because of the shape of the optimum value curves, the next method attempted was multiple polynomial regression. The resulting rule is given by

$$
\begin{aligned}
& I_{i}=1.5741+0.8985 \cdot\left(T_{h i}-300\right)-0.1529 \cdot\left(T_{c i}-300\right) \\
& -0.0047 \cdot\left(T_{h i}-300\right)^{2}+.0002 \cdot\left(T_{c i}-300\right)^{2} .
\end{aligned}
$$

A comparison of the optimum values of current and the values of current computed with

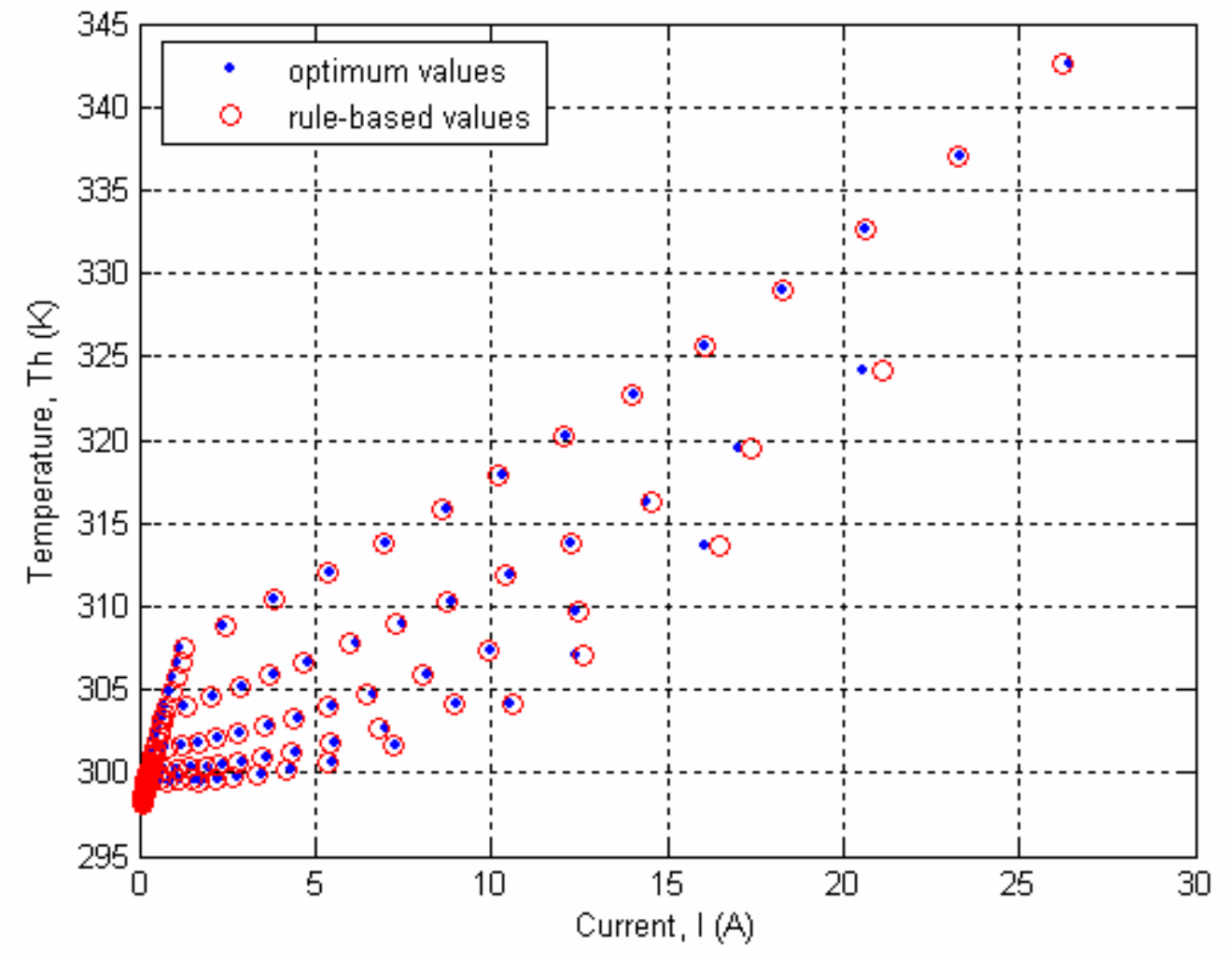

Figure 16. A plot of the optimum $C O P$ data (dots) compared to the data calculated from the distributed control rule (circles) as calculated using multiple polynomial regression (both $T_{c i}$ and $T_{h i}$ terms are squared). 
(19) is given in Figure 16. In this figure, the rule-based values closely match the optimum values with only a few dots being plotted outside of the resulting circles. Examining (19) shows that the final term influences the result very little. Comparing the partial derivatives of (19) shows that the $T_{c i}$ squared term affects the result less than 5\% of the $T_{h i}$ squared term. In order to simplify the control of the TEC, the distributed control rule should contain as few terms as possible while closely approximating the optimum values. The last method uses the multiple polynomial regression technique as before but drops the $T_{c i}$ squared term. The resulting rule is given by

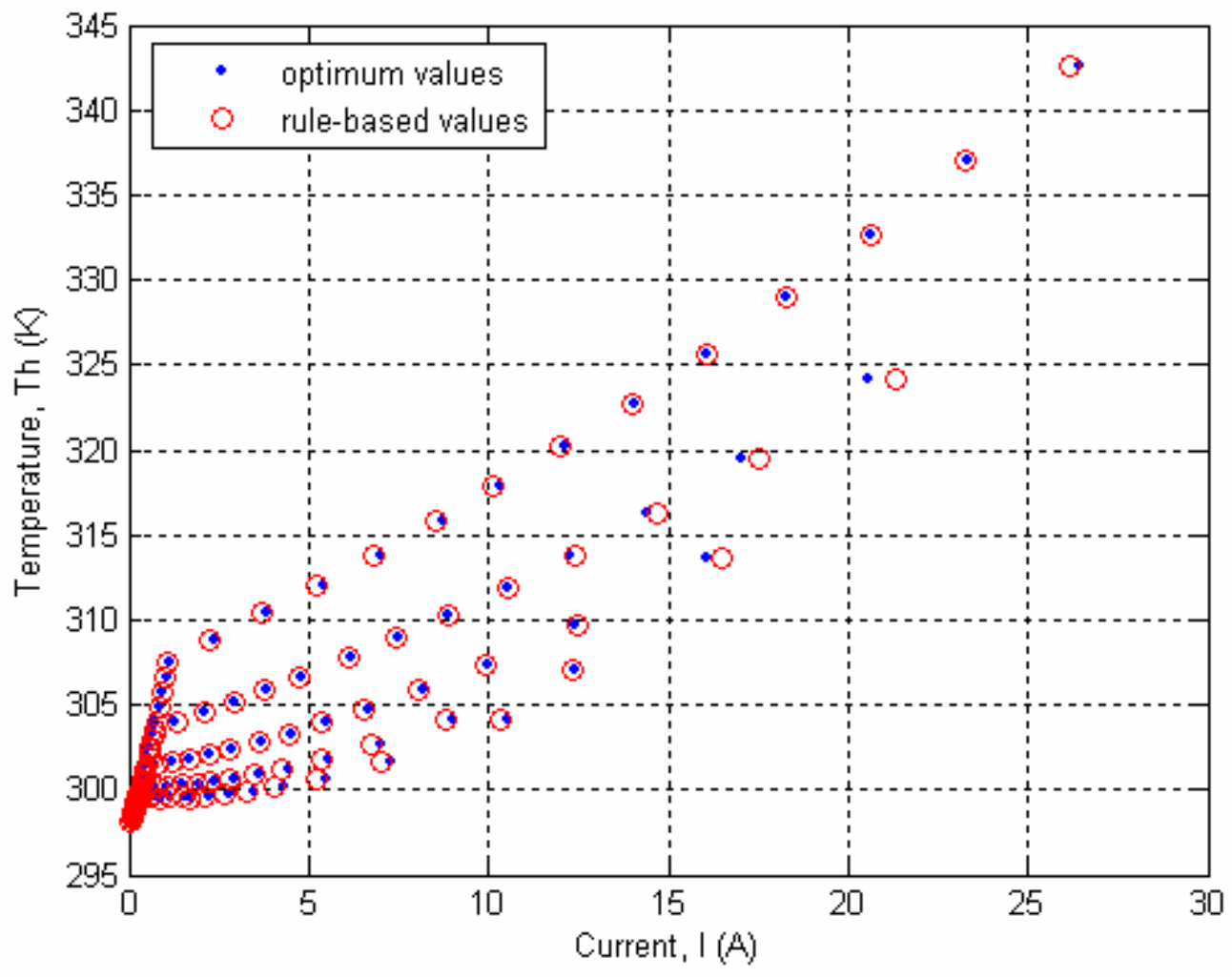

Figure 17. A plot of the optimum $C O P$ data (dots) compared to the data calculated from the distributed control rule (circles) as calculated using multiple polynomial regression ( $T_{h i}$-squared term only). 


$$
I_{i}=1.5877+0.9126 \cdot\left(T_{h i}-300\right)-0.1491 \cdot\left(T_{c i}-300\right)-0.0050 \cdot\left(T_{h i}-300\right)^{2},
$$

and the results are plotted in Figure 17. The results show that the $T_{h i}$-squared term is adequate to retain the shape of the curve and the accuracy of the distributed control rule. Based on the number of terms and closeness of fit, (20) will be used as the distributed control rule for the remainder of the results given in this work. The resulting $C O P$ values from applying (20) are also plotted in Figure 14 as the rule-based values. The rule-based values are just slightly less than the optimum values throughout the entire operating regime. Note that the rule-based values still represent a considerable improvement over the serial values. Each computed value differs by less than $1.0 \mathrm{~A}$ from the optimum value, and, in most cases, by less than $0.1 \mathrm{~A}$. These minor variations in the computed

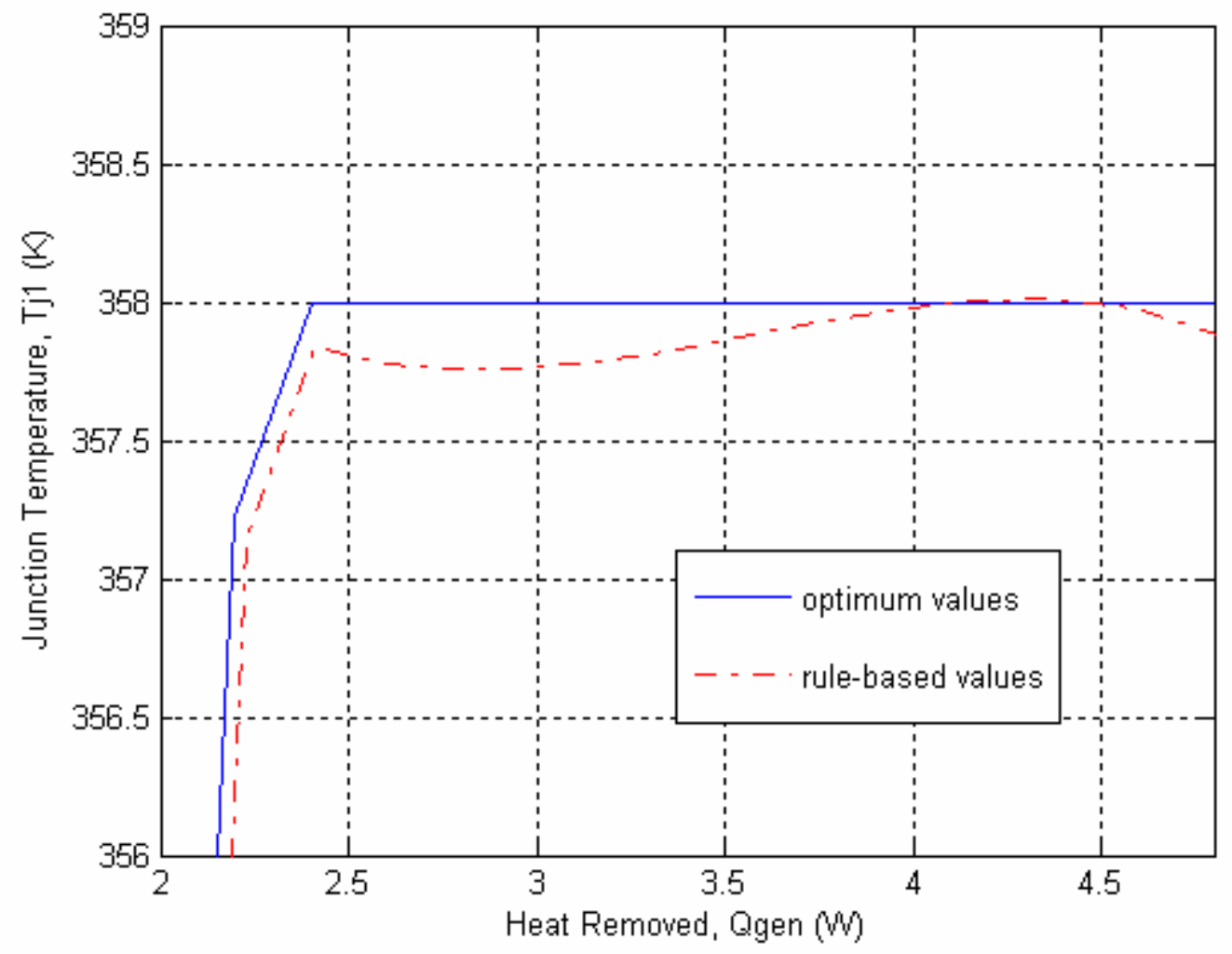

Figure 18. Plots of the optimum $T_{j l}$ and $T_{j l}$ calculated from the distributed control rule versus the heat removed by the TEC. 


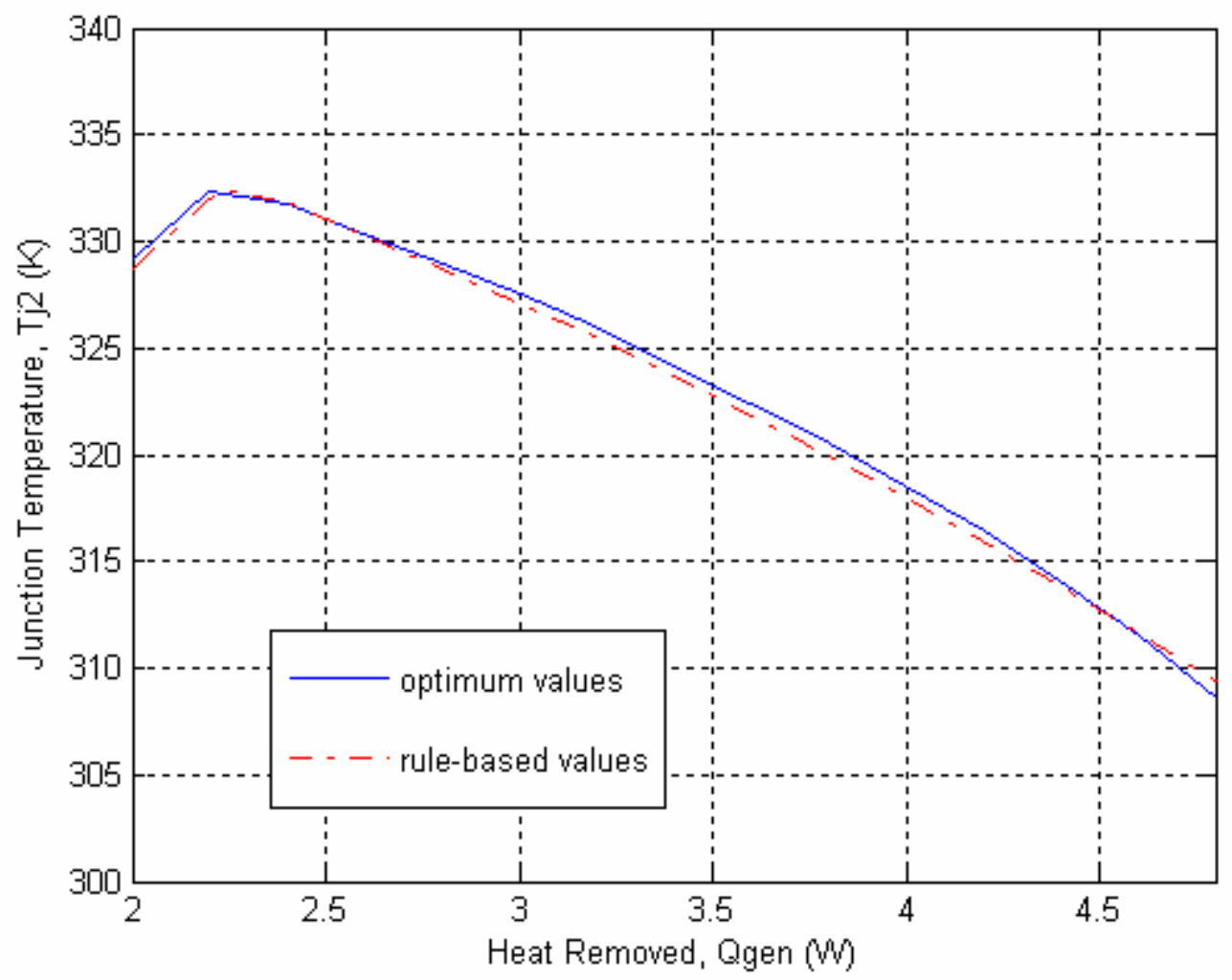

Figure 19. Plots of the optimum $T_{j 2}$ and $T_{j 2}$ calculated from the distributed control rule versus the heat removed by the TEC.

values from the optimum values do affect the $C O P$ adversely as shown in Figure 14. However, the reduction is minimal compared to the benefit gained over the serial value. To ensure the new scheme does not enter a forbidden operating regime, the junction temperatures, $T_{j i}$, were plotted for the optimum current values and the rule-based current values. The resulting graph for the first thermocouple is presented in Figure 18. The resulting graph for the second thermocouple is presented in Figure 19. For the optimum values, the junction temperature for the first thermocouple rises steadily until it reaches the maximum allowable junction temperature, $T_{\text {jmax }}$. At this point, approximately $2.4 \mathrm{~W}$, the couple must be powered to maintain $T_{\text {jmax }}$ throughout the operating regime. The rule- 
based value closely follows the optimum values. The junction temperature, $T_{j l}$, remains just below the $T_{j \max }$ throughout most of the operating regime. Although $T_{j l}$ does barely exceed $T_{\text {jmax }}$ between 4.0 and $4.5 \mathrm{~W}$, this minor breach should be inconsequential.

Figure 18 shows that the rule-based computations can stay within system limitations and produce the desired gains.

In Figure 19, the second junction temperature, $T_{j 2}$, initially rises due to the heat from conduction, $Q_{\text {condl }}$. At approximately $2.4 \mathrm{~W}$, the second thermocouple starts to consume more current and actually starts to lower $T_{j 2}$. Recall that this is the same heat generation, $Q_{\text {genl }}$, which the first couple must respond in order to maintain $T_{j l}$ less than $T_{j \max }$. This reduction in temperature at first may seem counterintuitive to the goal of increasing $C O P$, since $T_{j 2}$ is not approaching $T_{j \max }$. However, the benefit in this reduction is to increase the temperature difference between $T_{j l}$ and $T_{j 2}$, thereby increasing $Q_{c o n d l}$ as shown in (10). So, the second couple, as well as the other couples, gradually assumes more of the heat load as it increases, although another portion of the chip is producing the heat. The benefit of each couple assuming a portion of the heat load can be seen in the Joule heating term of (6). Each couple consumes a small amount of current to optimize the load on the primary thermocouple, thereby controlling the Joule heating. Figure 20 illustrates the degree to which the five thermocouples assume three different heat loads. Also shown, are the corresponding $C O P \mathrm{~s}$.

The assumption of heat load by the other couples will be proportional to the lateral thermal resistance between the portions of the chip, $R_{\text {cond }}$. As $R_{\text {cond }}$ approaches infinity, the assumption of heat load by the other couples will approach zero. As discussed for the two-thermocouple analysis this limit will require each thermocouple to 


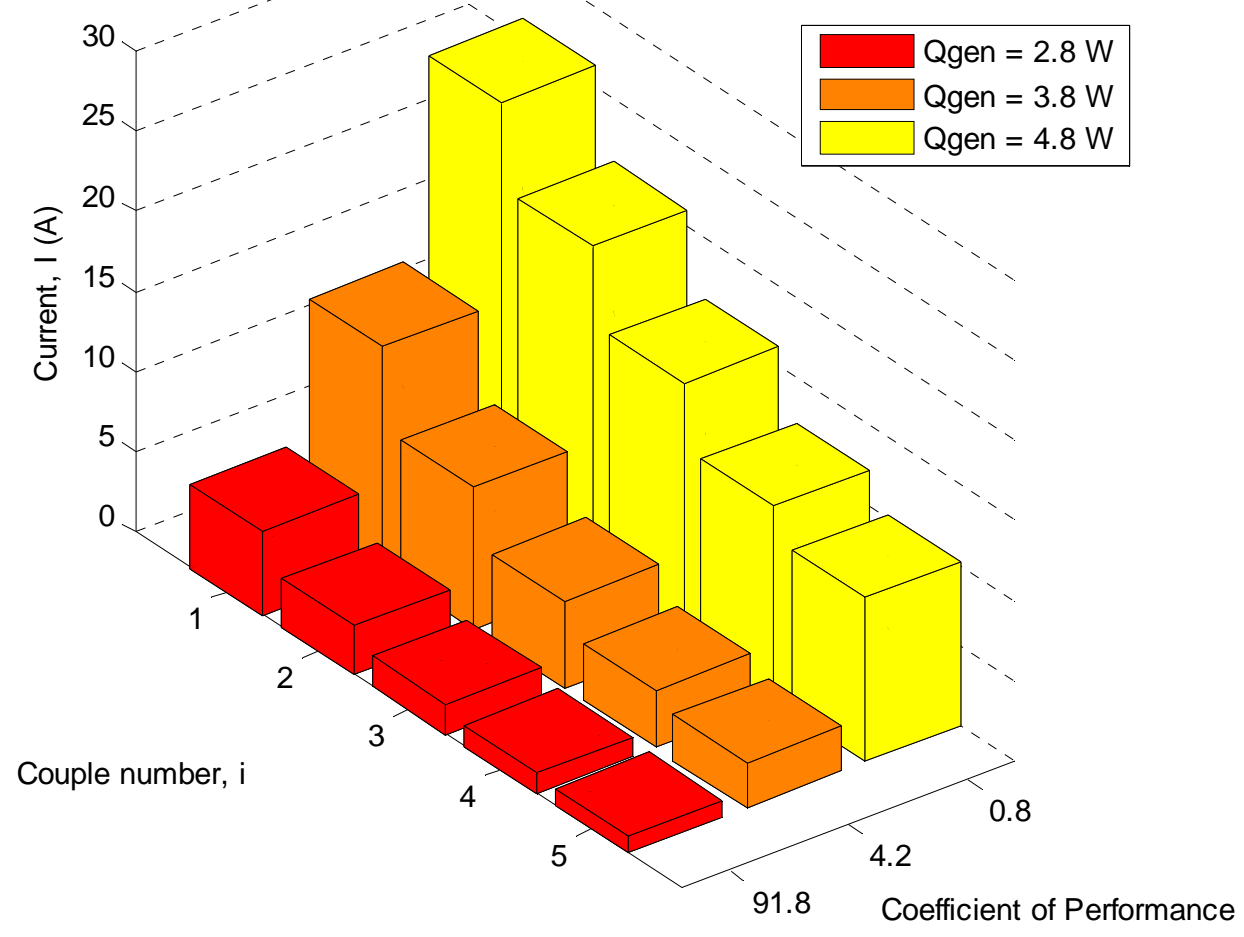

Figure 20. The assumption of three different heat loads and the corresponding COPs for the five-thermocouple model.

manage the heat load of its assigned chip portion alone. This means that the assigned chip portion can only produce an amount of heat less than or equal to the capacity of the individual thermocouple. As $R_{\text {cond }}$ approaches zero, the assumption of heat load will approach a uniform distribution of heat load. Again, in this limit, distributed control offers no benefit. The optimum solution will be the same as a serially connected TEC. This relationship shows that a heat spreader with a high thermal conductivity may benefit a traditional TEC. However, a TEC operated with distributed control will benefit from a reduced value of thermal conductivity and should not employ a heat spreader. 


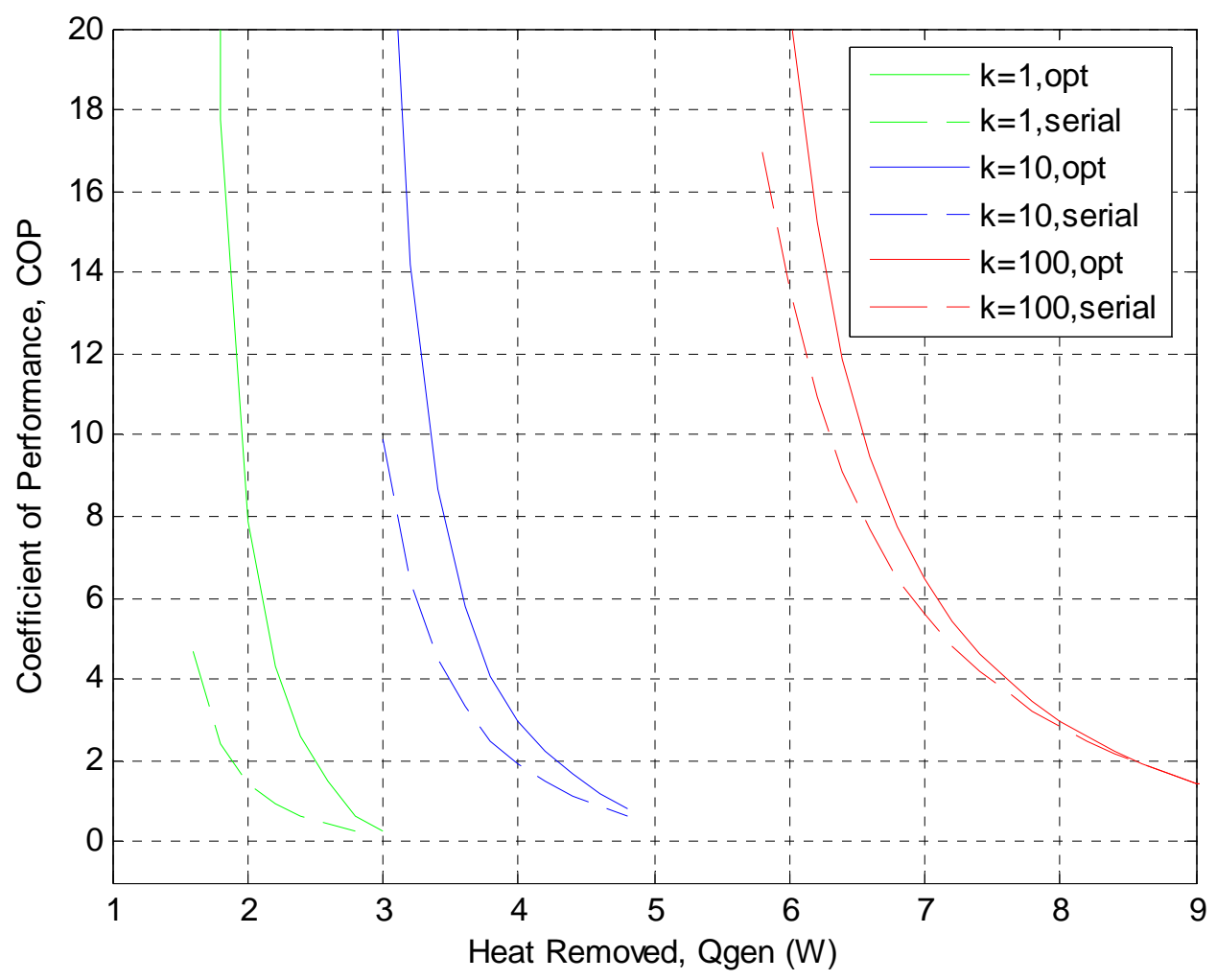

Figure 21. Comparison of optimum COPs for the distributed control case (solid curves) and the $C O P$ s for the serial case (dashed curves) for different values of lateral conductivity, $k_{\text {cond }}$. The analysis in this paper uses $k_{\text {cond }}=10 \mathrm{~W} / \mathrm{mK}$ corresponding to the middle set of plots.

Figure 21 compares the $C O P$ s for the serial and distributed control cases for three different values of lateral conductivity, $k_{\text {cond }}$. Lateral conductivity is inversely proportional to lateral thermal resistance, $R_{\text {cond }}$. The figure shows the gain from distributed control as the space between the solid and the dashed curves. As the value of $k_{\text {cond }}$ increases, the benefit that distributed control offers decreases. The middle set of plots represents the results presented in this paper. These results imply that the performance can be modified based on the material, and its conductivity, chosen as a heat spreader. 
One additional characteristic to be considered is the effect of the thermal conductivity of interfaces on the performance. The values of $R_{1}$ and $R_{2}$ were selected to be representative of the average conditions of existing systems examined. These values do change based on the variance in contact resistances, TIM, and fin-fan systems. Figure 22 shows how the $C O P$ varies over a wide range of $R_{2}$ values with $R_{1}$ held constant for an intermediate heat load of $3.9 \mathrm{~W}$. The optimum, the rule-based, and the serial values are shown. $R_{2}$ was chosen to vary because it typically has the widest range of values. The figure shows that the optimum $C O P S$ represent a two-fold increase in serial $C O P$ values for $3.9 \mathrm{~W}$ regardless of the $R_{2}$ value. The most significant feature in Figure 22 is that the

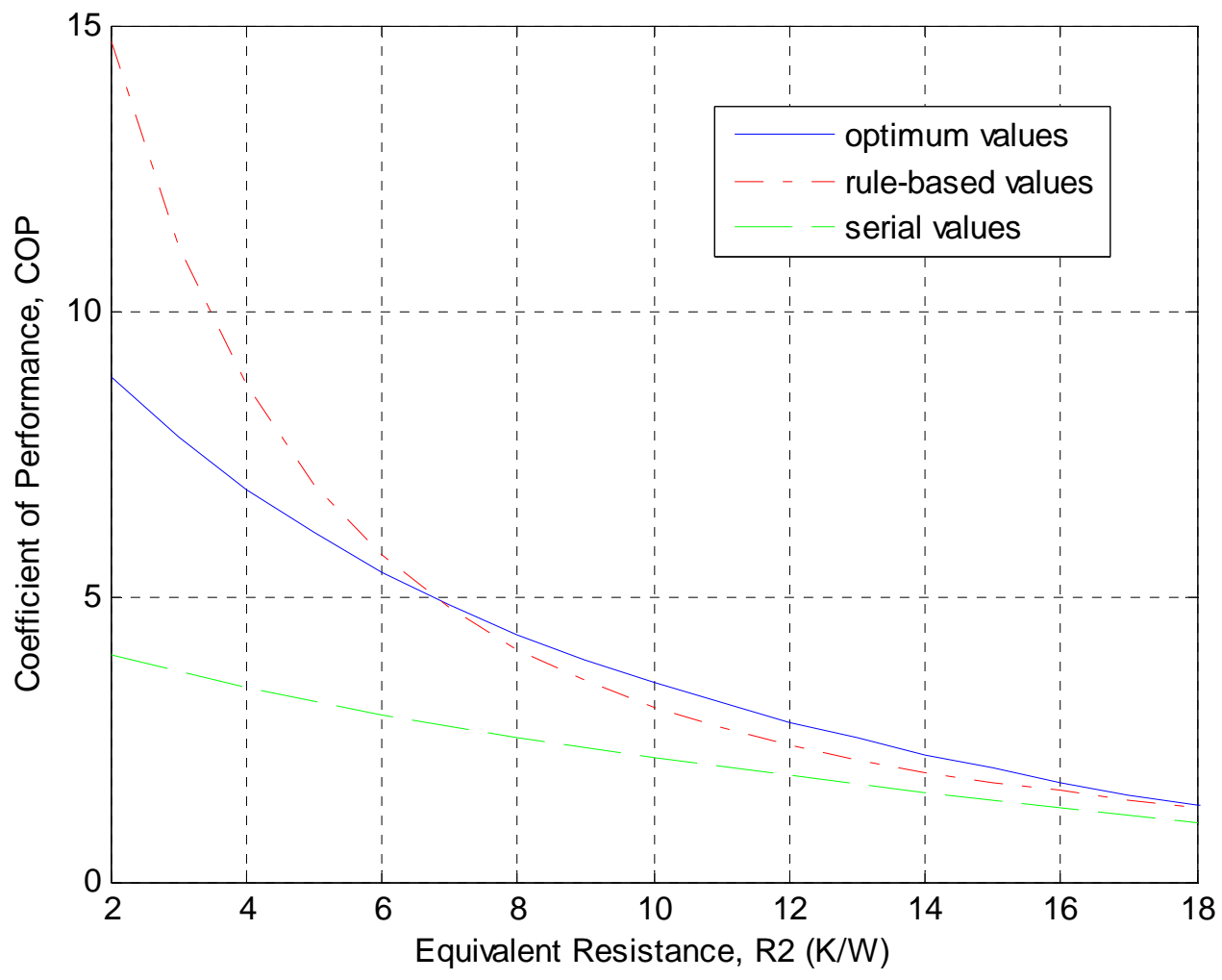

Figure 22. Variation of COP over a wide range of $R_{2}$ values with a constant value of $R_{1}$ and a constant heat load of $3.9 \mathrm{~W}$. 
rule-based values exceed the optimum values. This is not possible and represents a breakdown of the distributed control rule. The distributed control values were calculated using the same method as before, but the resulting values do not adequately represent the optimum values. The graph in Figure 22 essentially shows values in the region where COP becomes erratic. The values are erroneous and represent parameters in a forbidden regime. One theory for this behavior is that for small values of $R_{2}$, slight deviations in current results in drastic changes of other parameters that the model cannot predict. The distributed control rule holds up for small variances in $R_{2}$, but when $R_{2}$ varies considerably from those used in this paper a new method must be used to calculate the rule. 


\section{CHAPTER IV}

\section{CONCLUSIONS}

\section{Summary}

The need for improved performance of TECs is well documented. Current TECs are operated electrically in series and suffer from low efficiencies. Because of this efficiency problem and the ever-increasing heat removal demands of computer processors, a breakthrough in cooling performance is vital. Many research efforts are focusing on material advances, but distributed control offers an alternative to controlling TECs that produces significant improvements. By managing the individual thermocouples internal to a typical TEC with distributed control, significant gains in performance can be realized. A computer model was developed that determines the optimum performance of the individual thermocouples in a simplified linear array for a nonuniform heat distribution case. This model is scalable and can be used to analyze increasingly complicated scenarios to better determine feasibility of this technology.

By applying a distributed control rule to the TEC, it has been shown that the optimum performance can be closely approximated. This rule accepts the sensed local temperature values and determines the current loads to individual thermocouples to approximate maximum efficiency. The results represent improved performance throughout the operating regime by an average of $80 \%$ over a typical serial type TEC. In much of the regime, this improvement is 2.0 times the traditional values. This

improvement could, as a minimum, narrow the gap between computing technology and 
cooling capability. The benefits found here can be used in conjunction with any material advances to produce compounded improvements in performance. Distributed control of thermoelectric devices for chip-cooling applications where non-uniform heating occurs is a technology that deserves further investigation. The results presented here indicate an enhanced performance from this novel approach.

In addition to obtaining a control rule, the effects of changes in effective thermal resistances were examined. The potential benefits of distributed control increase as lateral conduction is reduced. This result may be exploited and preclude the requirement for a heat spreader. For changes in contact resistances, the potential benefits of distributed control are consistent, although the rule must allow for a system's particular parameters.

\section{Future Work}

Many directions are available to extend this work. The model itself can be extended to include more couples and to examine various heat distributions. The model could also be expanded to two dimensions. Eventually scaling the model to be on the order of an actual TEC and to be applied to actual processor architectures would be beneficial. This final step in the modeling process would require in-depth investigation of system parameters such as effective thermal resistances. Some considerations must be given to interconnect heating as well. Due to the increased number of connections required by distributed control, the amount of power losses due to interconnect heating will also increase. Preliminary calculations show that due to the high current levels and the small sizes, the worst-case scenario of individual control of each couple may be 
prohibitive. One solution may be offered by controlling the individual couples in clusters or arrays. For instance, by controlling the thermocouples in rows, no additional interconnects would be needed internal to the TEC. Another example would be to match the size of the cluster to the different areas of operation in the chip architecture. These prospects must be investigated to a greater extent to determine feasibility.

Another immediate issue affecting feasibility of distributed control is thermal fatigue. The application of distributed control will reduce temperature gradients across the computer chip, and therefore, reduce thermal stresses internal to the chip itself. However, current TEC technology is quite susceptible to thermal fatigue due to cycling. Traditional TECs are powered at one level constantly, and are not frequently cycled. Frequent cycling of a TEC causes the internal electrical connections to fail. Some advances in materials and manufacturing have already been considered and some have been implemented [43]. The extent of the thermal stresses due to distributed control and their effects should also be a future consideration.

Finally, lab experiments must be conducted to validate the model. Initially, these experiments may be conducted by controlling currents manually and measuring parameters to calculate COPs. Eventually, feedback sensors and actuators should be used to examine system response and viability. 


\section{APPENDIX A}

\section{SAMPLE COMPUTER CODE}

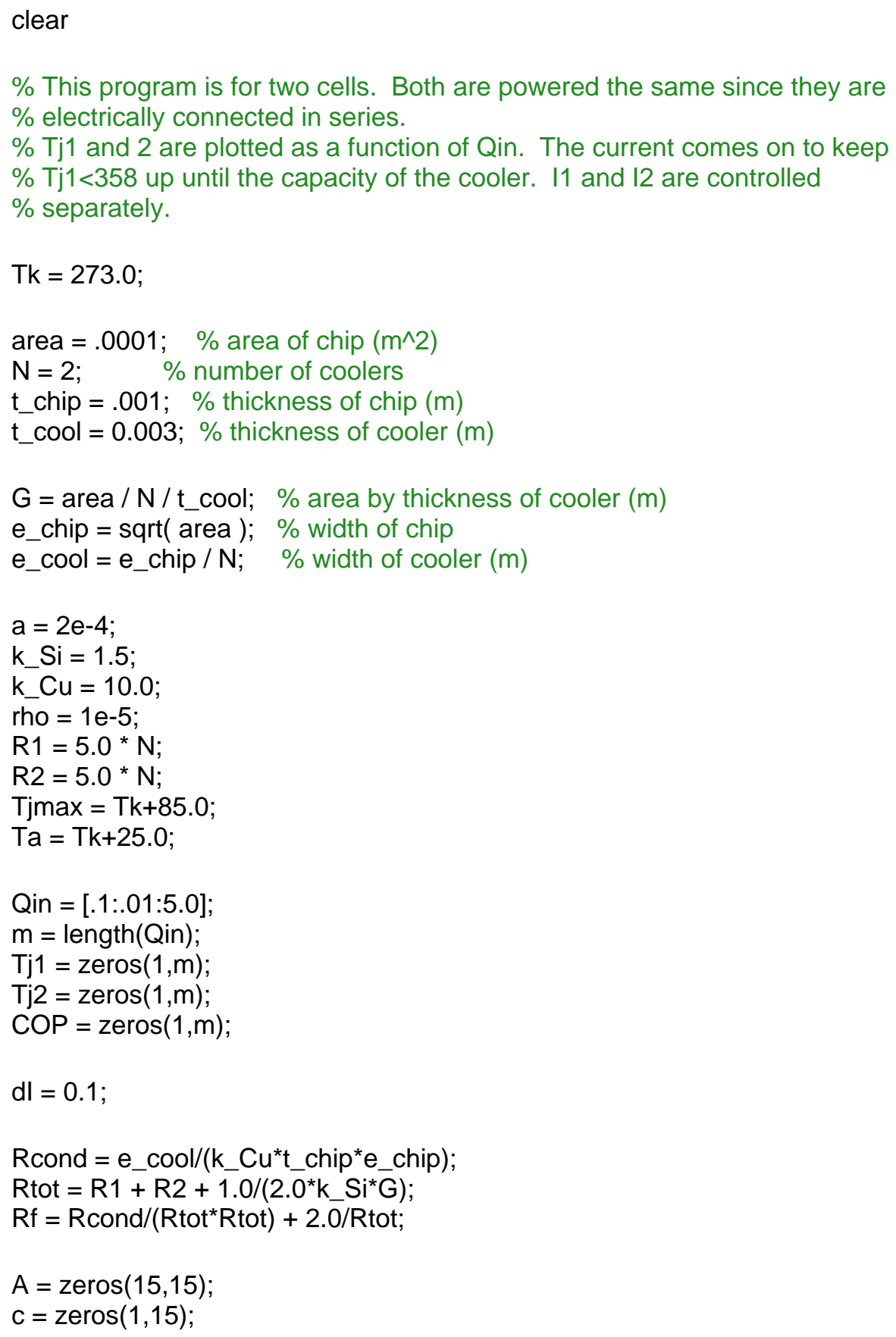




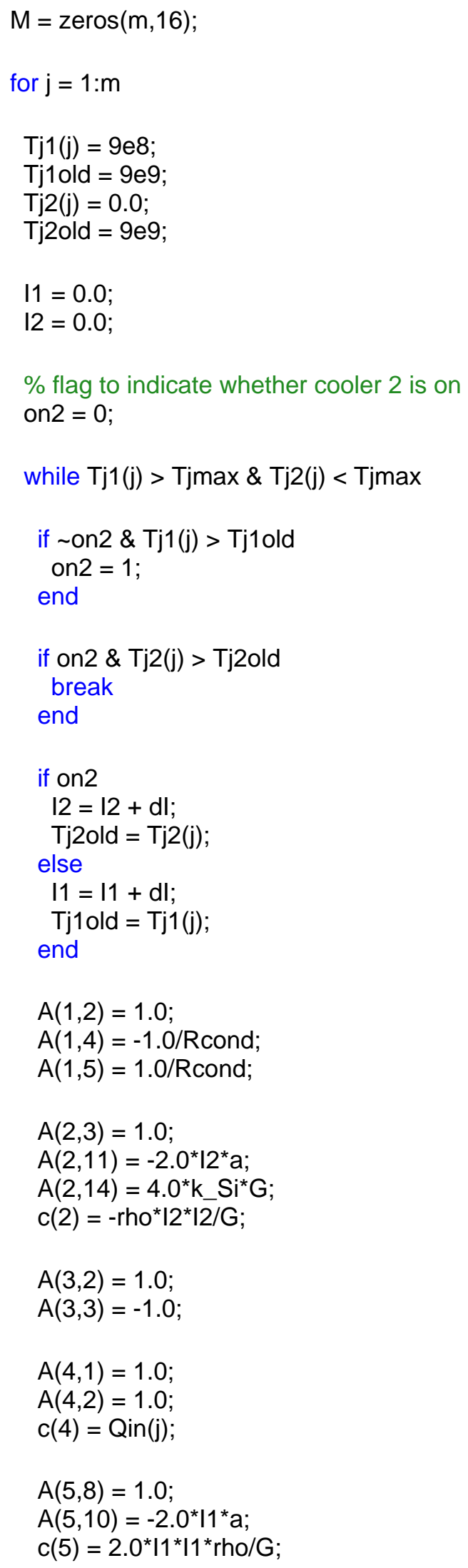




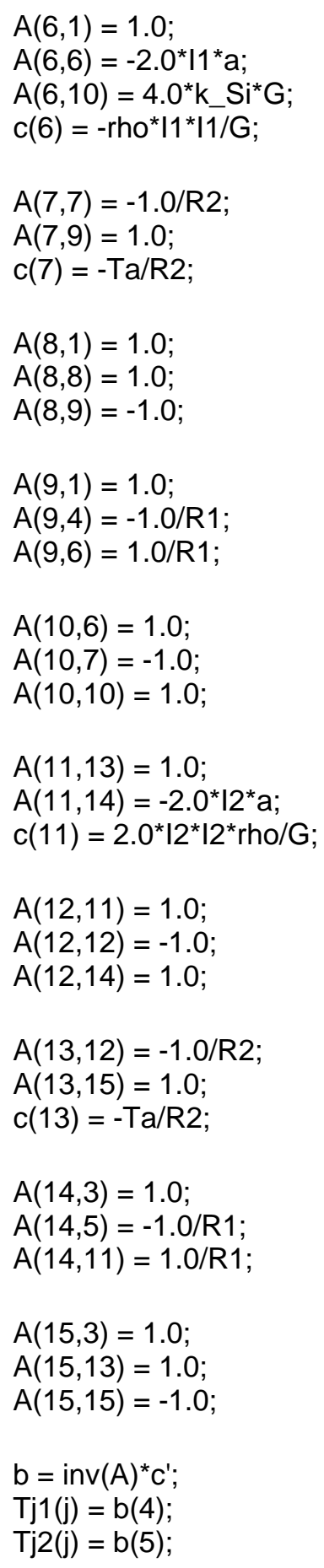

end

Qnom $=\left(\mathrm{Tj} 1(\mathrm{j})+\mathrm{Tj} 2(\mathrm{j})-2^{\star} \mathrm{Ta}\right) /\left(\mathrm{R} 1+\mathrm{R} 2+2^{*} \mathrm{k} \_\mathrm{Si}^{\star} \mathrm{G}\right)$;

$\operatorname{COP}(j)=(b(1)+b(3)) /(b(8)+b(13))$;

$\operatorname{Inhh}(\mathrm{j})=\mathrm{I} 1$;

$12 \mathrm{hhh}(\mathrm{j})=\mathrm{I} 2$;

$M(j, 1)=Q i n(j)$ 
$M(j, 2: 16)=b^{\prime} ;$

$\%$ sanity check

\%Qin(j) - b(1) - b(3)

end

save dump M -ascii

hold on

figure(1); plot(Qin, Tj1,'.',Qin, Tj2,'‘');

hold on

figure(2); plot(Qin(m-200:m-50),COP(m-200:m-50)); axis ([3.3 4.3 l 010$]$ );

hold on

figure(3); plot(Qin,I1hhh,'.',Qin,I2hhh,'*'); 


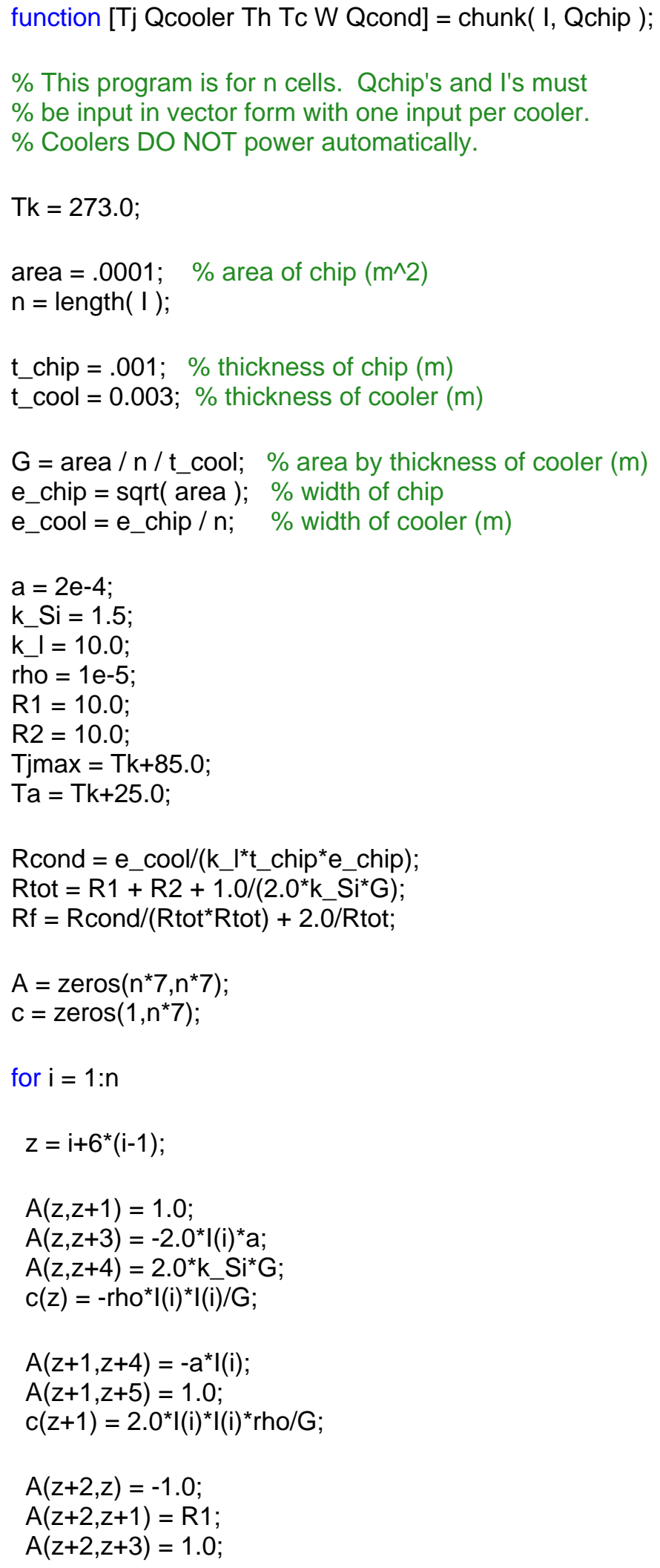




$$
\begin{aligned}
& A(z+3, z+2)=-1.0 ; \\
& A(z+3, z+3)=1.0 ; \\
& A(z+3, z+4)=1.0 ; \\
& A(z+4, z+1)=R 2 ; \\
& A(z+4, z+2)=-1.0 ; \\
& A(z+4, z+5)=R 2 ; \\
& c(z+4)=-T a ; \\
& A(z+5, z+1)=1.0 ; \\
& A(z+5, z+6)=1.0 ; \\
& \text { if } i>1 \\
& A(z+5, z-1)=-1.0 ; \\
& \% e l s e \\
& \% A(z+5, z+6)=0.0 \\
& \text { end } \\
& c(z+5)=Q c h i p(i) ; \\
& A(z+6, z)=-1.0 ; \\
& A(z+6, z+6)=R c o n d ; \\
& \text { if } i<n \\
& A(z+6, z+7)=1.0 ; \\
& \text { else } \\
& \text { A }(z+6, z)=0.0 ; \\
& \text { end } \\
& \text { end }
\end{aligned}
$$

end 


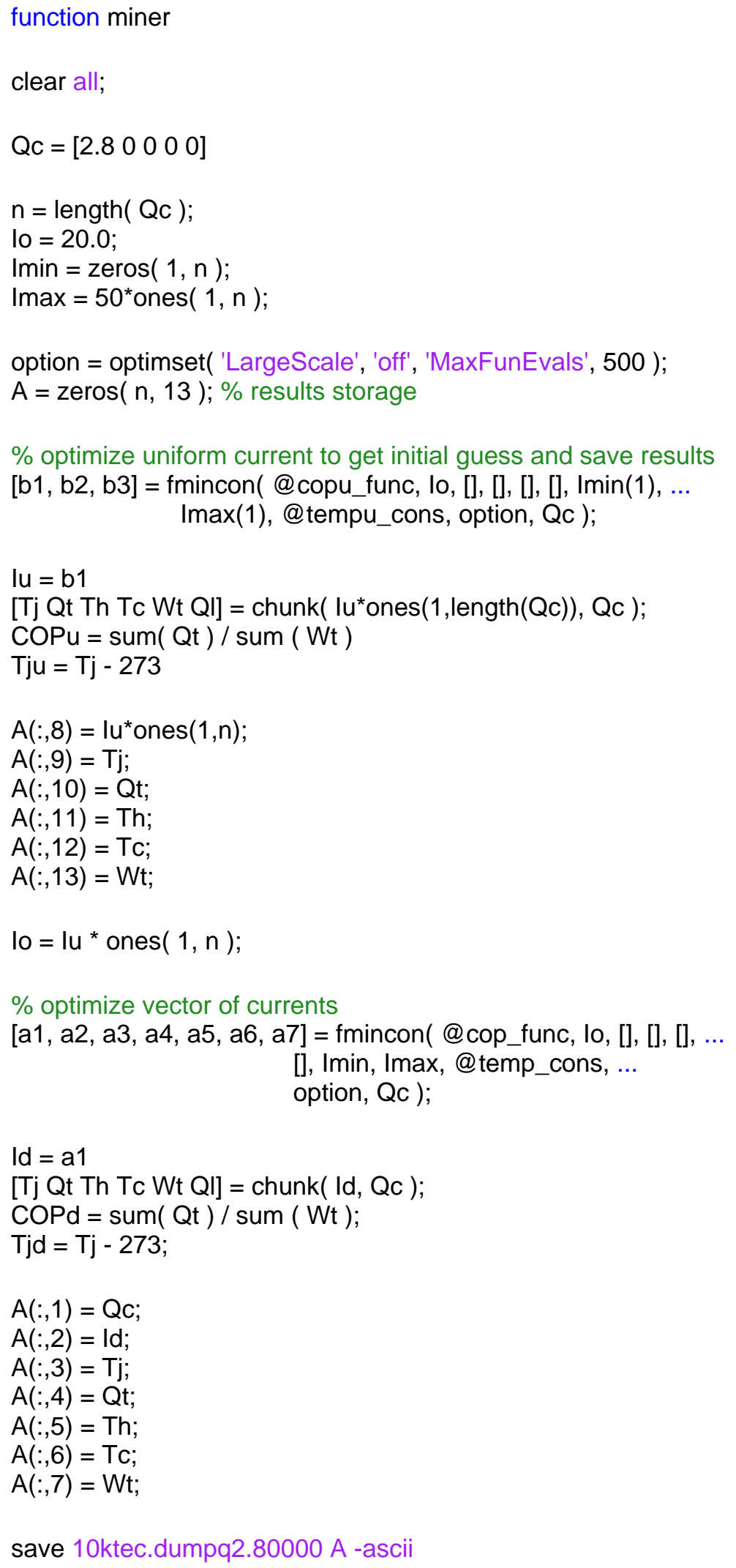




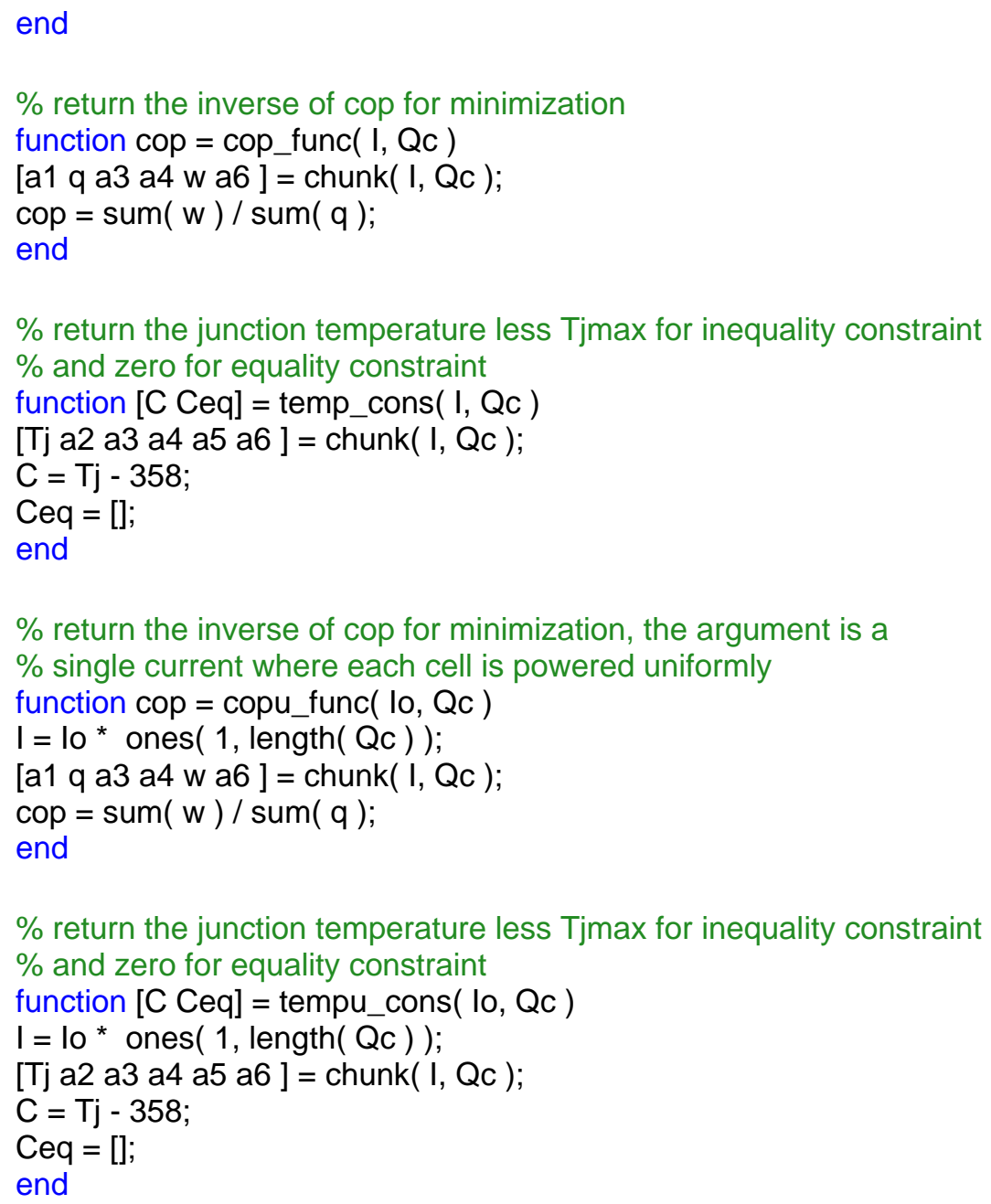




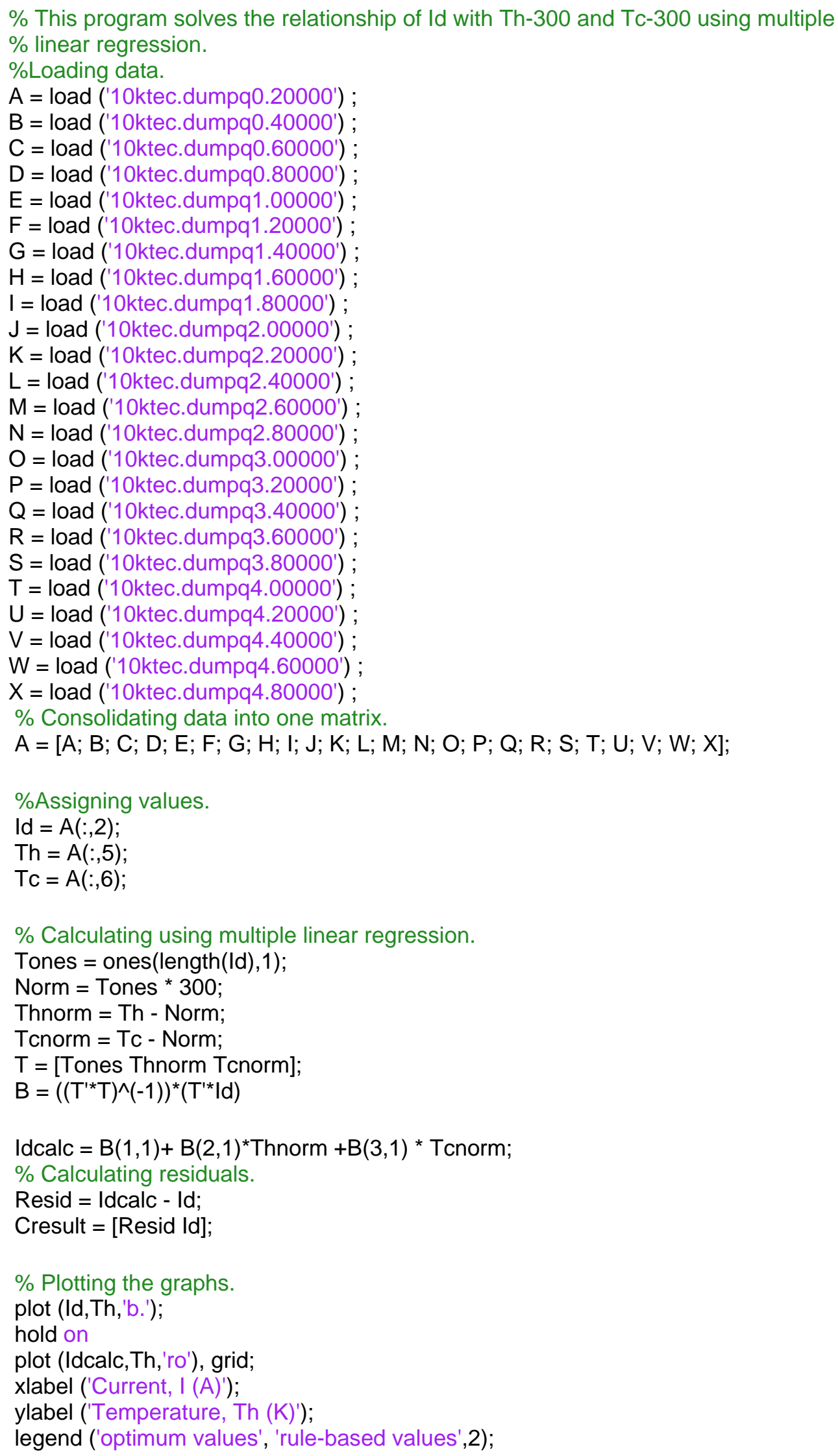




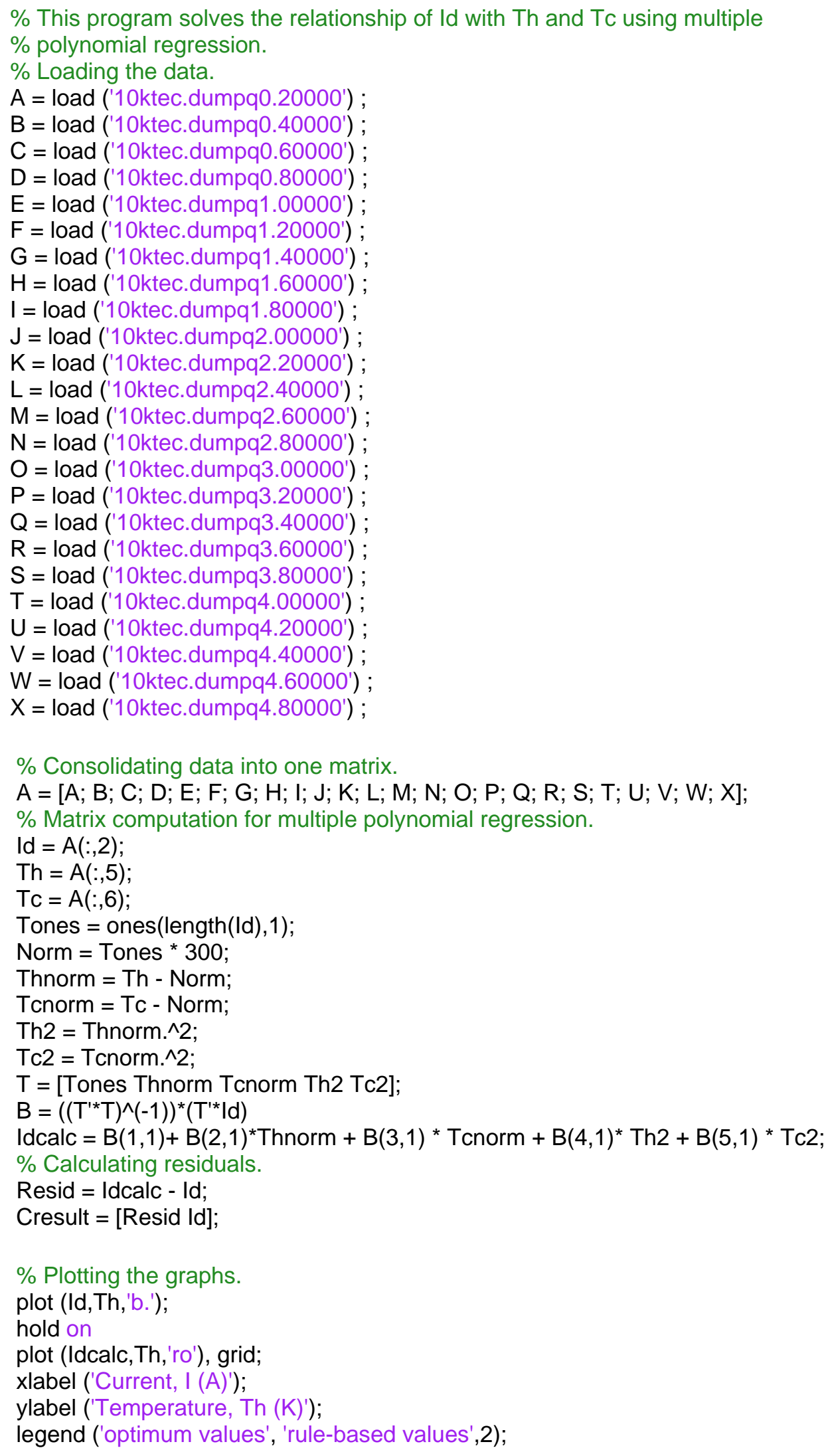




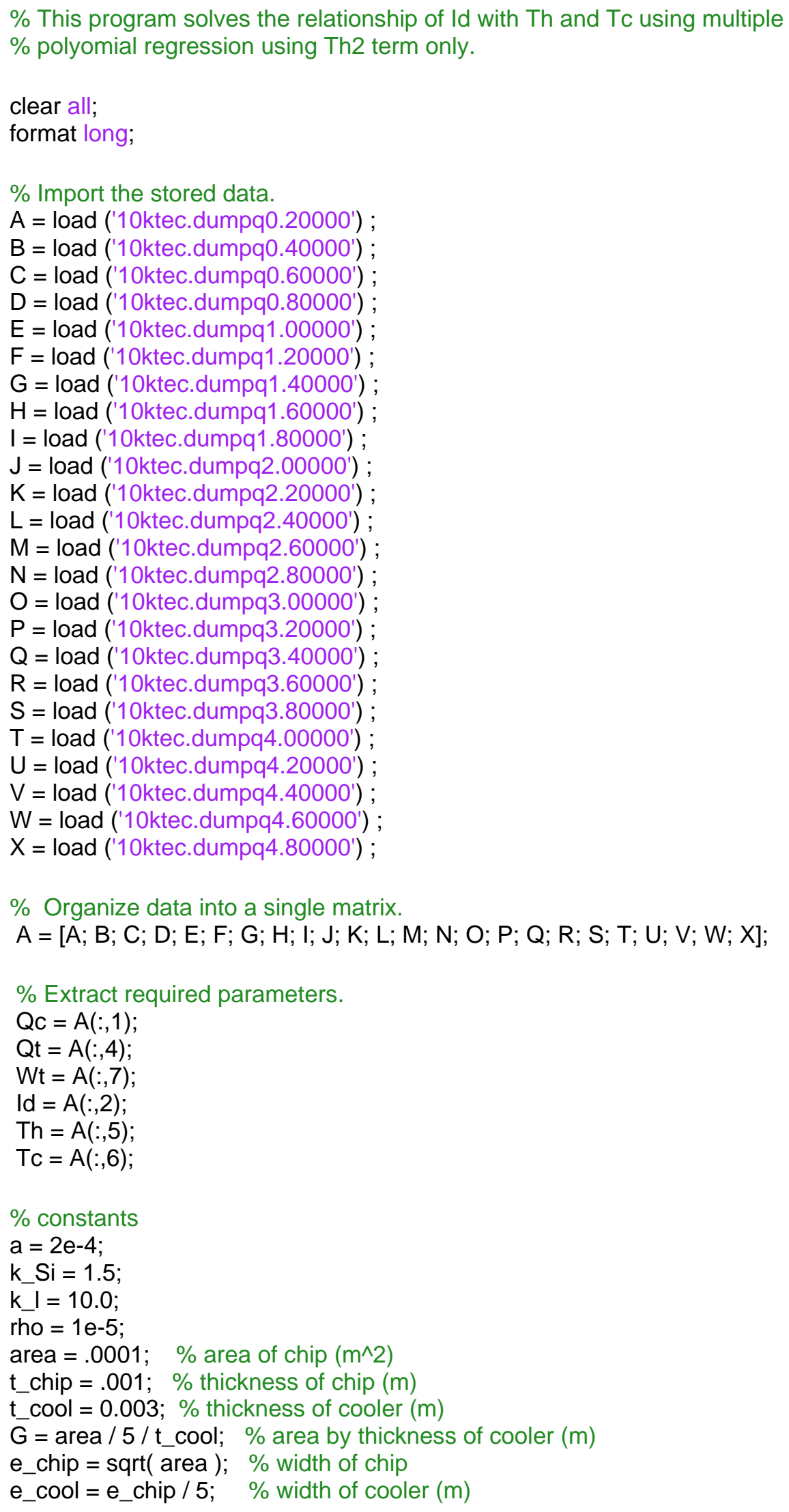




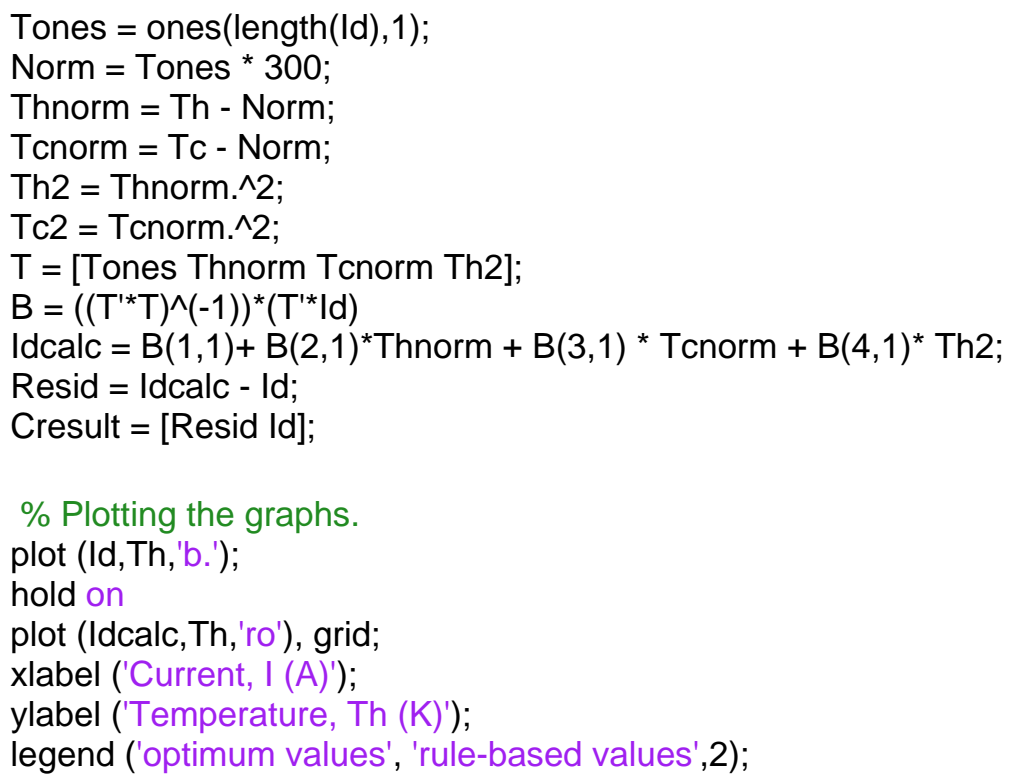




\section{REFERENCES}

[1] P. E. Phelan, V. A. Chirac, and T. -Y. T. Lee. Current and future miniature refrigeration cooling technologies for high power microelectronics. IEEE Transactions on Components and Packing Technologies, 25(3):356-365, September 2002.

[2] H.J. Goldsmid. A review of new thermoelectric materials. IEEE $20^{\text {th }}$ International Conference on Thermoelectrics, 2001.

[3] C. Uher. Skutterudites: promising power conversion thermoelectrics. IEEE $22^{\text {nd }}$ International Conference on Thermoelectrics, 2003.

[4] G. Chen, T. Zeng, T. Borca-Tasciuc, and D. Song. Phonon engineering in nanostructures for solid-state energy conversion. Material Science and Engineering, A292:155-161, 2000.

[5] J.W. Sharp, S.J. Poon, and H.J. Goldsmid. Boundary scattering and the thermoelectric figure of merit. Solid State Physics, 187:507-516, 2001.

[6] L.W. da Silva and M. Kaviany. Micro-thermoelectric cooler: interfacial effects on thermal and electrical transport. International Journal of Heat and Mass Transfer, 47:2417-2435, 2004.

[7] J. Nurnus, H. Böttner, C. Kunzel, U. Vetter, A. Lambrecht, J. Schumann, and F. Völklein. Thin film based thermoelectric energy conversion systems. IEEE $21^{\text {st }}$ International Conference on Thermoelectrics, 2002.

[8] G. Chen. Nanoscale heat transfer and nanostructured thermoelectrics. Inter Society Conference on Thermal Phenomena, pages 8-16, 2004.

[9] D. Astrain, J.G. Vián, and M. Domínguez. Increase of COP in the thermoelectric refrigeration by the optimization of heat dissipation. Applied Thermal Engineering, 23:2183-2200, 2003.

[10] S.B. Riffat and X. Ma. Improving the coefficient of performance of thermoelectric cooling systems: a review. International Journal of Energy Research, 28:75-768, 2004.

[11] X.C. Xuan. Investigation of thermal contact effect on thermoelectric coolers. Energy Conversion and Management, 44:399-410, 2003.

[12] J.P. Gwinn and R.L. Webb. Performance and testing of thermal interface materials. Microelectronics Journal, 34:215-222, 2003. 
[13] J. Chen, Y. Zhou, H. Wang, and J.T. Wang. Comparison of the optimal performance of single- and two-stage thermoelectric refrigeration systems. Applied Energy, 73:285-298, 2002.

[14] V. Semenyuk. Cascade thermoelectric micro modules for spot cooling high power electronic components. IEEE $21^{\text {st }}$ International Conference on Thermoelectrics, 2002.

[15] M. Hodes. One-dimensional analysis of thermoelectric modules. IEEE Inter Society Conference of Thermal Phenomena, 2004.

[16] The International Technology Roadmap for Semiconductors, Semiconductor Industry Association, 2004 update edition.

[17] A. Majumdar. Thermoelectricity in semiconductor nanostructures. Science, 303:777-778, 6 February 2004.

[18] A. Shakouri. Nanoscale devices for solid state refrigeration and power generation. 20th IEEE Semi- Therm Symposium, 2004.

[19] R. F. Service. Temperature rises for devices that turn heat into electricity. Science, 306:803-807, 29 October 2004.

[20] D. G. Walker, K. D. Frampton, and R. D. Harvey. Distributed control of thermoelectric coolers. Inter Society Conference on Thermal Phenomena, pages 361-366, 2004.

[21] R. S. Viswanath, V. S. Wakharkar, A. Watwe, and V. Lebonheur. Thermal performance challenges from silicon to systems. Intel Technology Journal, Q3 2000 .

[22] F. Giulietti, L. Pollini, and M. Innocenti. Autonomous formation flight. IEEE Control Systems Magazine, pages 34-44, December 2000.

[23] M. Kader, M. Lenczner, and Z. Mrcarica. Distributed control based on distributed electronic circuits: application to vibration control. Microelectronics Reliability, 41:1857-1866, 2001.

[24] R. D. Harvey, D. G. Walker, and K. D. Frampton. Distributed control to improve performance of thermoelectric coolers. IMECE, 2004.

[25] K. Chen and G.T. Lin. Optimization of multiple-module thermoelectric coolers using artificial-intelligence techniques. International Journal of Energy Research, 26:1269-1283, 2002. 
[26] H.J. Goldsmid. Principles of thermoelectric devices. British Journal of Applied Physics, 11:209-217, June 1960.

[27] H.J. Goldsmid and R.W. Douglas. The use of semiconductors in thermoelectric refrigeration. British Journal of Applied Physics, pages 386-390, 1954.

[28] H. J. Goldsmid. Thermoelectric Refrigeration. Plenum Press, New York, 1964.

[29] M.V. Vedernikov and E.K. Iordanishvili. A.F. Ioffe and origin of modern semiconductor thermoelectric energy conversion. $17^{\text {th }}$ International Conference on Thermoelectrics, pages 37-42, 1998.

[30] H.J. Goldsmid. Timeliness in the development of thermoelectric cooling. $17^{\text {th }}$ International Conference on Thermoelectrics, pages 25-28, 1998.

[31] J.C. Bass, D.T. Allen, S. Ghamaty, and N.B. Elsner. New technology for thermoelectric cooling. 20 th IEEE SEMI-THERM Symposium, 2004.

[32] H.J. Goldsmid. Possibilities of improvement in thermoelectric refrigeration. $18^{\text {th }}$ International Conference on Thermoelectrics, pages 531-535, 1999.

[33] H.J. Goldsmid. Solid-state and vacuum thermoelements. $22^{\text {nd }}$ International Conference on Thermoelectrics, pages 433-438, 2003.

[34] B.C. Sales. Smaller is cooler. Science, 295:1248-1249, 15 February 2002.

[35] F. J. DiSalvo. Thermoelectric cooling and power generation. Science, 285:703706, July 1999.

[36] T.C. Harman, P.J. Taylor, M.P. Walsh, and B.E. LaForge. Quantum dot superlattice thermoelectric materials and devices. Science, 297:2229-2232, 27 September 2002.

[37] A. Khitun, A. Balandin, J.L. Liu and K.L. Wang. The effect of the long range order in a quantum dot array on the in-plane lattice thermal conductivity. Superlattices and Microstructures, 30, No. 1, 2004.

[38] G. Chen, A. Narayanaswamy, and C.Dames. Engineering nanoscale phonon and photon transport for direct energy conversion. Superlattices and Microstructures, 35:161-172, 2004.

[39] V. Semeniouk and J.P. Fleurial. Novel high performance thermoelectric microcoolers with diamond substrates. $16^{\text {th }}$ International Conference on Thermoelectrics, pages 683-686, 1997. 
[40] P.K. Schelling, L. Shi, and K.E. Goodson. Managing heat for electronics. Materials Today, pages 30-35, June 2005.

[41] G.J. Snyder. Design and optimization of compatible, segmented thermoelectric generators. $22^{\text {nd }}$ International Conference on Thermoelectrics, pages 443-446, 2003.

[42] A.G.A. Nnanna. Application of refrigeration system in electronics cooling. Applied Thermal Engineering, Article in press, 2005.

[43] Y. M. Hoi, D.D.L. Chung, Flexible graphite as a compliant thermoelectric material. Letters to the Editor / Carbon, 40:1131-1150, 2002. 